\title{
Locative and Directional Prepositions in Conceptual Spaces: The Role of Polar Convexity
}

\author{
Joost Zwarts ${ }^{1}$ • Peter Gärdenfors ${ }^{2,3}$
}

Published online: 22 September 2015

(C) The Author(s) 2015. This article is published with open access at Springerlink.com

\begin{abstract}
We approach the semantics of prepositions from the perspective of conceptual spaces. Focusing on purely spatial locative and directional prepositions, we analyze both types of prepositions in terms of polar coordinates instead of Cartesian coordinates. This makes it possible to demonstrate that the property of convexity holds quite generally in the domain of prepositions of location and direction, supporting the important role that this property plays in conceptual spaces.
\end{abstract}

Keywords Prepositions $\cdot$ Polar coordinates $\cdot$ Conceptual spaces $\cdot$ Convexity

\section{Semantic Constraints on Spatial Prepositions}

Prepositions are a limited class of words, but with a wide range of meanings and uses, even if we consider only locative prepositions in one language, like English in, near, over or behind (Lindstromberg 2010).

(1) a. There is a fly in my soup.

b. The bomb went off near a guest house.

c. Install the door knob over the key slot.

d. Keep behind the line.

$凶$ Joost Zwarts

j.zwarts@uu.nl

Peter Gärdenfors

Peter.Gardenfors@lucs.lu.se

1 Utrecht Institute of Linguistics, Utrecht University, Utrecht, The Netherlands

2 Cognitive Science, Department of Philosophy, Lund University, Lund, Sweden

3 Social Robotics Studio, Centre of Quantum Computation and Intelligent Systems, University of Technology, Sydney, Australia 
These locative prepositions define a relation between a trajector and landmark, by identifying a particular 'region of acceptability' or 'search domain' with respect to the landmark where the trajector can be found (Logan and Sadler 1996; Levinson 1996). Different geometries can be the basis of a preposition's meaning, like topology or projective geometry (Crangle and Suppes 1989) with different frames of reference, making use of deictic, intrinsic or environmental features (Levinson 1996). Various non-geometric factors (like function and force-dynamics) also play an important role (e.g. Herskovits 1986; Vandeloise 1991; Bowerman 1996; Feist and Gentner 1998; Coventry and Garrod 2004; Carlson and van der Zee 2005; Gärdenfors 2014).

Since prepositions are at the crossroads of spatial cognition, linguistic expression, and computational application, this variety and flexibility has been the topic of many theoretical and experimental studies by psychologists, linguists, and information scientists, within and across languages. This research has increased our understanding of the many factors that play a role in prepositional meanings and the way this might differ between languages. ${ }^{1}$

However, this also raises the much less investigated question whether and how meanings of prepositions are constrained, that is, how all the different spatial and other factors come together in such a way that they can be learned, represented, and used by human beings. Although this is a general question about word meanings, it is especially relevant for prepositions, given their importance at many different levels of lexicon, grammar, and discourse. Landau and Jackendoff (1993), Regier (1996), and Zwarts and Winter (2000) pay attention to the constraints on prepositional meanings from different perspectives. In this article, we approach this issue from the perspective of conceptual spaces, with a focus on the geometric constraint of convexity (Gärdenfors 2000,2014 ), which has already been shown to be at work in a range of different domains. In this approach, word meanings correspond to convex regions (subsets of points) in a conceptual space, which are defined as follows (Gärdenfors 2000, p. 69):

(2) A subset $C$ of a conceptual space $S$ is said to be convex if, for all points $x$ and $y$ in $C$, all points between $x$ and $y$ are also in $C$.

One concrete example of the constraint of convexity is found in the domain of color words. Jäger (2010) has shown that for the languages included in the World Color Survey there is strong evidence that color words correspond to convex regions of the color space.

Although applying convexity to locative prepositions might seem a simple matter, it is far from trivial, because it depends heavily on the assumptions that we make about the structure and representation of space. Although definition (2) suggest that there is a straightforward and intuitive notion of convexity, we believe that the semantics of prepositions shows that convexity depends on the way spatial positions are represented in relation to a particular origo. Our goal in this paper is therefore not empirical, but theoretical: building on what is known about prepositions, we will show that, when

\footnotetext{
1 The literature is too large and varied to do justice to, but the number of recent monographs and volumes in this domain illustrate the interest in prepositions and spatial language (Levinson 2003; Coventry and Garrod 2004; Carlson and van der Zee 2005; Levinson and Wilkins 2006; Saint-Dizier 2006; Asbury et al. 2008; Mani and Pustejovsky 2012; Vulchanova and van der Zee 2012).
} 
using polar coordinates, a particular version of convexity holds quite generally in the domain of prepositions and it can serve as a guide to alternative semantic analyses in those cases where it does not seem to hold.

The structure of the paper is as follows. Section 2 briefly sketches the background of conceptual spaces in relation to the semantics of prepositions. We then spell out our model of polar coordinates in Sect. 3, which we use to define the meanings of locative prepositions in Sect. 4. In Sect. 5 we demonstrate to what extent the resulting meanings are convex with respect to a new notion of polar betweenness. We extend our model to directional prepositions in Sect. 6.

\section{Conceptual Spaces, Convexity, and Locative Prepositions}

In order to model the meaning of prepositions we need to make some general assumptions, first about the role of regions and convexity in conceptual spaces and then about the way prepositions are treated.

As we already saw, an important notion in the semantic theory of Gärdenfors (2000, 2014 ) is that of convexity, the thesis that concepts can be represented as convex regions of some conceptual space. A fact that is often overlooked is that there are several ways of conceiving of betweenness. In traditional Euclidean geometry, the points between the points $a$ and $c$ are the points that lie on the straight line between $a$ and $c$. For a 3-dimensional Cartesian space with coordinates $x, y$ and $z$, the notion of betweenness can be more formally defined as follows:

(3) A point $b=\left\langle x_{b}, y_{b}, z_{b}\right\rangle$ lies between a point $a=\left\langle x_{a}, y_{a}, z_{a}\right\rangle$ and a point $c=$ $\left\langle x_{c}, y_{c}, z_{c}\right\rangle$ if there is some $k, 0<k<1$ such that $x_{b}=k x_{a}+(1-k) x_{c}$, $y_{b}=k y_{a}+(1-k) y_{c}$ and $z_{b}=k z_{a}+(1-k) z_{c}$.

As we will see, however, using polar coordinates, another notion of betweenness can be defined and thereby another notion of convexity.

Spatial prepositional phrases (PPs) like in my soup, near a guest house, over the key slot, and behind the line can also be taken to correspond to regions in a conceptual space, physical space in this case. What kind of points do these regions consist of? One idea is that the region corresponding to a spatial PP consists of a set of vectors. The use of vectors in the semantics of prepositions has been advocated from different points of view: to account for their relation with cognitive maps (O'Keefe 1996, 2003), for the way they are modified (e.g. three feet above, right behind, Zwarts 1997; Zwarts and Winter 2000), the role that attention plays in their use (Regier and Carlson 2001), for modeling their frames of reference and points of view (Bohnemeyer 2012), and their relation with syntactic structures (Svenonius 2006, 2008; Kracht 2008). The notion of a vector is a simple but powerful way to represent the position of the trajector relative to the landmark. Its starting point is somewhere in the landmark and its end point is somewhere in the trajector and the preposition imposes restrictions on the length and direction of the vector. A prepositional phrase like below the lamp corresponds then to the set of vectors that have their origin in the lamp and that point downward, each representing a potential position for a trajector in that region. We assume that all prepositions that have a spatial, geometric meaning can be represented using vectors in this way. 
However, there are also many prepositions, some of them quite basic, for which it has been argued that they are not based on geometric factors, but on force-dynamic or functional factors (for example, Herskovits 1986; Vandeloise 1991; Dewell 1994; Bowerman 1996; Garrod et al. 1999; Tyler and Evans 2001; Beliën 2002; Coventry et al. 2001; Coventry and Garrod 2004; Carlson and van der Zee 2005; Zwarts 2010; Gärdenfors 2014). There is debate in the literature about whether prepositions like at, in and on, and over and under are defined entirely in terms of non-geometric factors, or whether their meaning involves a combination of geometric and functional elements. Here we follow Gärdenfors (2014) in assuming that these prepositions are not spatial in nature, but force-dynamic. We apply our analysis to locative prepositions that are more clearly spatial (although functional factors can also play a role for these prepositions too): the topological prepositions inside and outside, the distance prepositions near and far, the projective (or axial) prepositions above, below, in front of, behind, beside, and the preposition between. In Sect. 6, we then turn to directional prepositions, like to, from, and through.

\section{Spatial Representation Using Polar Coordinates}

Most commonly, space is represented with the aid of the Cartesian coordinates $x, y$ and $z$, representing 'width', 'depth' and 'height' and where distances are measured using a Cartesian metric. However, there is another way of representing space, namely in terms of polar coordinates, which represent points in space in terms of distance and angles.

It is important to note that we are not analyzing the metaphysics of space, but how humans cognitively represent space. We operate with several perspectives on space, the most important being the egocentric, where space is seen from the point of view of the speaker, and the allocentric, where space is seen "from outside". An interesting question, that seems to have been scarcely addressed, is what coordinate system best represents our spatial cognition. It seems plausible that our brains actually uses both types of coordinates (see Gallant et al. 1993 for evidence that some cells in macaque brains code for polar coordinates and some for Cartesian coordinates).

Let us present how a three-dimensional space $S$ can be defined in terms of polar coordinates, or more precisely, spherical coordinates (e.g. Marsden and Tromba 1981, p. 44). It is assumed that the space has an origo point $o$ and two axes $x$ and $z$. As illustrated in Fig. 1, a point $p$ is represented as a vector relative to that origo and those axes $x$ and $z$ as a triple $\langle r, \theta, \varphi\rangle$ :

(4) $r$ (the radius) is a real number (with $r \geq 0$ ) representing the distance of $p$ from the origo $o$,

$\theta$ (the azimuth angle) is the angle (with $0^{\circ} \leq \theta<360^{\circ}$ ) between $p$ and the $x$-axis, $\varphi$ (the polar angle) is the angle (with $0^{\circ} \leq \varphi \leq 180^{\circ}$ ) between $p$ and the positive $z$-axis (the zenith).

Notice that when the polar angle $\varphi$ is $0^{\circ}$ (straight 'up') or $180^{\circ}$ (straight 'down'), then the value of $\theta$ is arbitrary and that $\theta$ and $\varphi$ are both arbitrary when $r=0$, that is, at the origo. We assume that the angle $\theta$ goes counterclockwise when seen from the positive side of the $z$-axis (from 'above'), so that the negative $y$-axis corresponds 
Fig. 1 Representation of point $p$ in polar coordinates $\langle r, \theta, \varphi\rangle$. "3D Spherical 2" by Dmcq-Own work. Licensed under CC BY-SA 3.0 via Wikimedia Commons-https:// commons.wikimedia.org/wiki/ File:3D_Spherical_2.svg\#/ media/File:3D_Spherical_2.svg

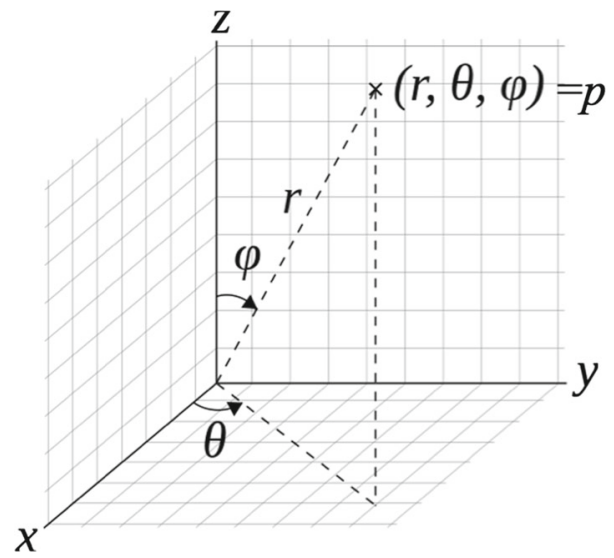

to $\theta=90^{\circ}$ and the positive $y$-axis to $\theta=270^{\circ}$. In geographical applications of polar coordinates, the $x$-axis represents 'north' and the $z$-axis represents 'up', as the fixed reference directions relative to which other angles are defined. We will not make such an assumption, but assume that the $x$-axis and $z$-axis are fixed in an arbitrary way, independently of linguistically relevant directions like 'up', and introduce such directions explicitly in Sect. 4.

We can assign to every pair of points $a$ and $b$ the shortest angle $\angle a b$ (in degrees) between them. ${ }^{2}$ This angle is $0^{\circ}$ for points that have the same angular coordinates. It is $180^{\circ}$ for points $a$ and $-a$ that are opposite and $90^{\circ}$ for points that are perpendicular to each other. These notions will come in useful in Sect. 4.

Given the representation of polar coordinates, one can define a notion of polar betweenness (p-betweenness) that is different from the one generated by the standard Cartesian metric:

(5) A point $b=\left\langle r_{b}, \theta_{b}, \varphi_{b}\right\rangle$ lies polarly between (p-between) a point $a=\left\langle r_{a}, \theta_{a}, \varphi_{a}\right\rangle$ and a point $c=\left\langle r_{c}, \theta_{c}, \varphi_{c}\right\rangle$ if there is some $k, 0<k<1$ such that

$$
\begin{aligned}
& r_{b}=k r_{a}+(1-k) r_{c}, \\
& \theta_{b}=k \theta_{a}+(1-k) \theta_{c} \quad \text { iff }\left|\theta_{a}-\theta_{c}\right|<180^{\circ}, \quad \text { and } \theta_{b}=k \theta_{a}+(1-k)\left(\theta_{c}-360^{\circ}\right) \\
& \text { iff }\left|\theta_{a}-\theta_{c}\right|>180^{\circ} \text {, } \\
& \text { and } \\
& \varphi_{b}=k \varphi_{a}+(1-k) \varphi_{c} .
\end{aligned}
$$

The division into two cases in the criterion for $\theta_{b}$ derives from the intuition that p-betweenness can only be defined for the shortest distance along the $\theta$-dimension. Otherwise, any point would lie between any two points on a circle. For example, when $\theta_{a}=45^{\circ}$ and $\theta_{c}=270^{\circ}$, then the second part of the definition in (5), when applied with $k=0.5$, gives a $\theta_{c}$ coordinate $-22.5(=337.5)$, which is halfway between $45^{\circ}$ and $270^{\circ}$ taking the shortest way. Note that the definition leaves betweenness undefined

$\overline{2 \angle a b \text { can be defined using the law of cosines in spherical trigonometry. }}$ 


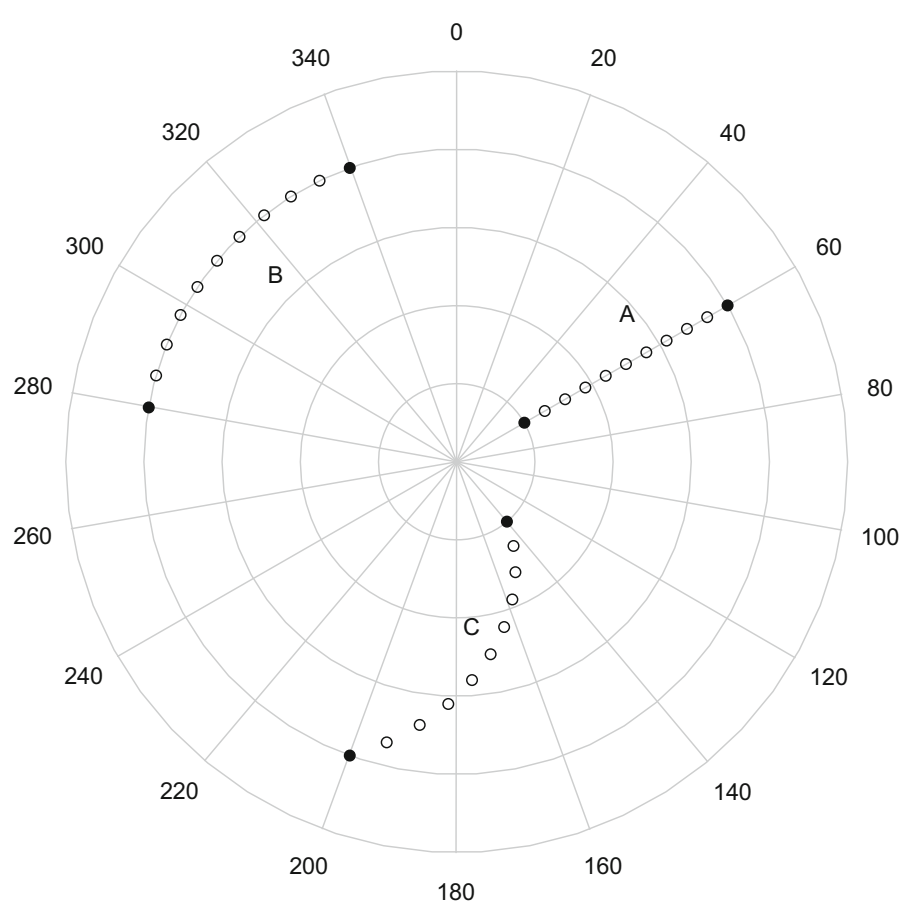

Fig. 2 Polar betweenness

in the case when $\left|\theta_{a}-\theta_{c}\right|=180^{\circ}$, when 'the shortest distance' between $\theta_{a}$ and $\theta_{c}$ is undefined. In order to apply the definition also for the case when $a$ (or $c$ ) is the origo, where the values of $\theta$ and $\varphi$ are undefined, we may put $\theta_{a}=\theta_{c}$ and $\varphi_{a}=\varphi_{c}$.

The polar coordinates introduce a different geometry on the space, compared with the standard Cartesian coordinates. Consequently, the 'line segments' generated by this polar betweenness relation can be 'curved', if seen with Cartesian glasses. ${ }^{3}$ This is illustrated in Fig. 2 for the two-dimensional case (omitting the polar angle $\varphi$ ) for three situations $\mathrm{A}, \mathrm{B}, \mathrm{C}$, in which the open circles are ' $b$ ' points p-between the ' $a$ ' and ' $c$ ' points represented by the black circles. The A situation shows that the points p-between $a$ and $c$ form a radial line if a and c differ only in radius. If they differ only in angle, then the points p-between them lie on a circle, as in situation B. If both angle and radius differ, then the p-between points describe a curve. To be more specific, in that case these points lie on an Archimedean spiral.

Two points at (almost) opposite sides of the origo are related by a curve that goes around the origo, as shown in Fig. 3. This illustrates the crucial difference with ordinary betweenness that will turn out to be relevant for the convexity of prepositions.

Consider now a region $R$ in $S$, represented as a set of points each defined as a polar coordinate with respect to one and the same origo $o$.

\footnotetext{
3 See Gärdenfors (2000, Section 3.5) for further details.
} 


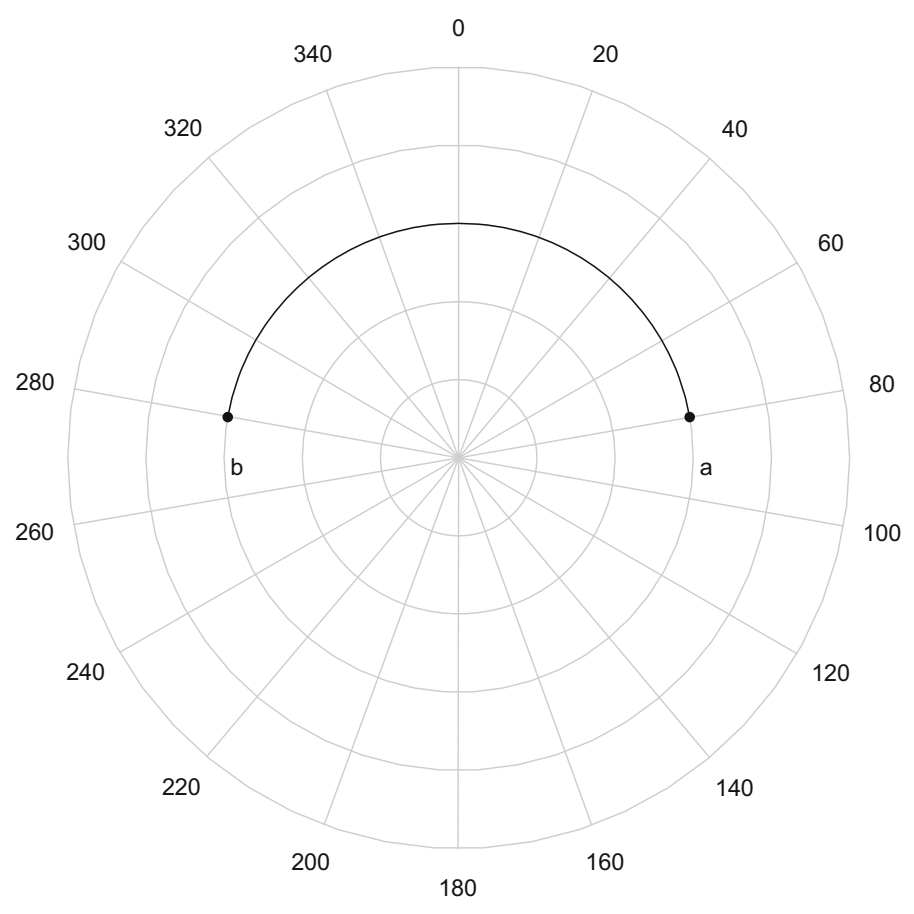

Fig. 3 P-betweenness around the origo

(6) A subset $R$ of $S$ is polarly convex (p-convex), if and only if, for all points $a$ and $c$ in $R$, any point $b$ in $S$ that is p-between $a$ and $c$ is also in $R$.

One thing to note about p-convexity, in comparison to Cartesian convexity, is under what coordinate transformations convexity is preserved. Cartesian convexity is preserved under multiplications, translations and rotations of the coordinate system. In contrast, polar convexity only preserves convexity under multiplications (changing the values of the $r$ coordinate) and rotations (changing the values of the $\varphi$ and $\theta$ coordinates). If a translation occurs, i.e. if the origo moves, then convexity may not be preserved. This underlines the important role that the origo plays in polar coordinate systems.

What kind of regions in three-dimensional space are p-convex? We will not give a full and systematic overview, but mention prominent examples that will be important later on for the p-convexity of prepositions (Sect. 5). A sphere is p-convex, but only if the origo of its polar coordinates lies in its center. The same is true for the interior of a sphere, its exterior, and the area between two spheres with different radii but the same origo. A full line or full plane is p-convex if it contains the origo. A half-line or line segment is p-convex if it is on the same line as the origo. A half-space or half-plane is p-convex if the origo is a point on the bounding plane or line. An infinite cone is p-convex with respect to its apex.

The intersection of two p-convex regions $R_{1}$ and $R_{2}$ is also p-convex, but only if the p-convexity of the two regions is based on the same origo. The intersection of 


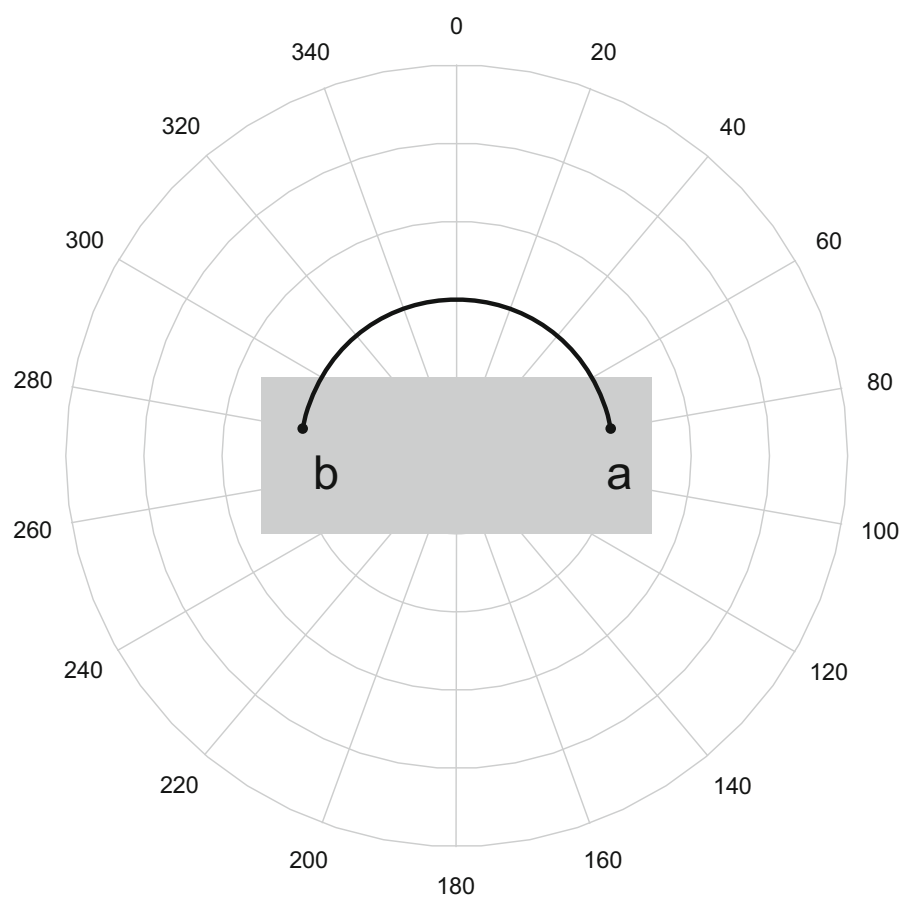

Fig. 4 Non-p-convexity of rectangle

a sphere with a half-space that goes through the origo is a hemisphere, for instance, which is also p-convex. Proof: Let $R_{1}$ and $R_{2}$ be two p-convex regions with the same origo and $a, c \in R_{1} \cap R_{2}$. Therefore, $a, c \in R_{1}$ and $a, c \in R_{2}$. Let $b$ be p-between $a$ and $b$. Then $b \in R_{1}$ and $b \in R_{2}$ because $R_{1}$ and $R_{2}$ are both p-convex. Therefore $b \in R_{1} \cap R_{2}$.

Regions with other familiar geometric shapes are not p-convex, even though they might be convex in terms of Cartesian coordinates, like a triangle, cube, or cylinder. A double cone is an example of a region that is neither convex in the Cartesian, nor in the polar sense. Suppose that $a$ and $b$ are points in the region of some of these shapes, then the curve of p-between points connecting $a$ and $b$ may not be entirely contained in the region, even if the origo $o$ has a central location. This is illustrated in Figs. 4 and 5.

The polar convexity defined above generalizes the two different continuity constraints that Zwarts (1997: 81) proposed for the region corresponding to a PP, and that are based on two different betweenness relations between non-zero vectors that are members of the same vector space. Roughly speaking, a vector $v$ is linearly between $u$ and $w$ if a situation as in the left part of Fig. 6 obtains and radially between two vectors $u$ and $w$ in a situation like in the right part (Zwarts 1997, p. 81).

A region $R$ corresponding to a locative PP is linearly (radially) continuous iff for every $u, w \in R$, if $v$ is linearly (radially) between $u$ and $w$, then $v \in R$. Zwarts argues that all locative PPs correspond to regions that are both linearly and radially continuous. Although he uses the term continuity, it is clear that these constraints 


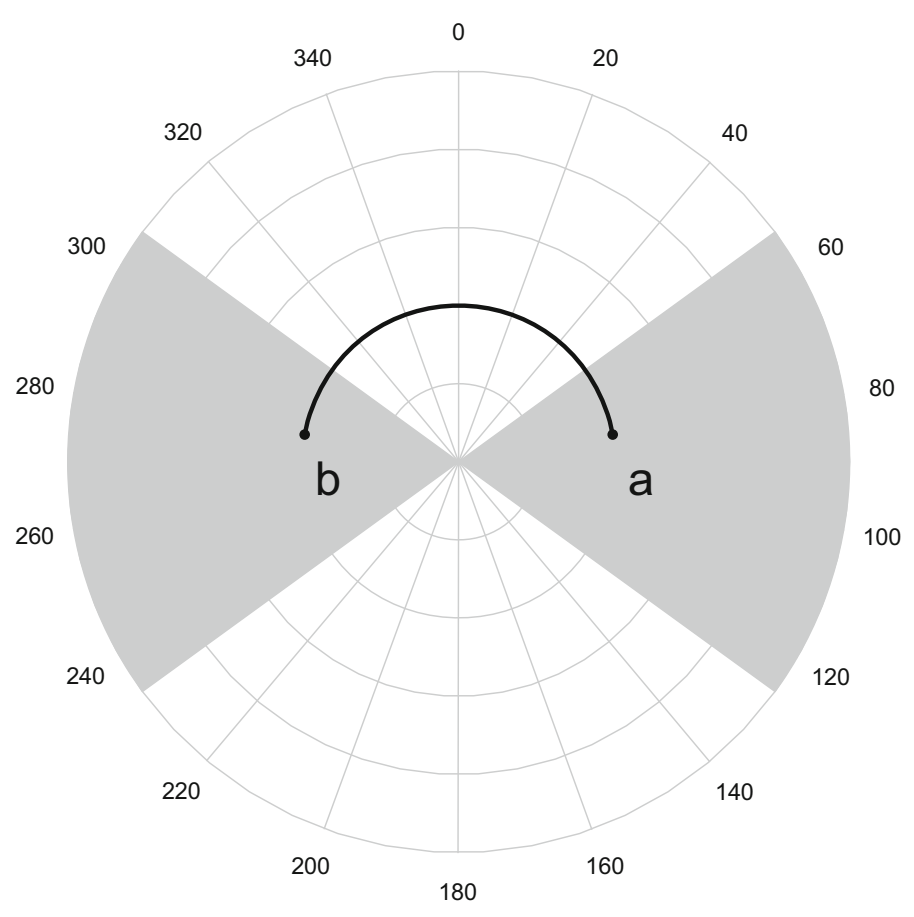

Fig. 5 Non-p-convexity of double cone
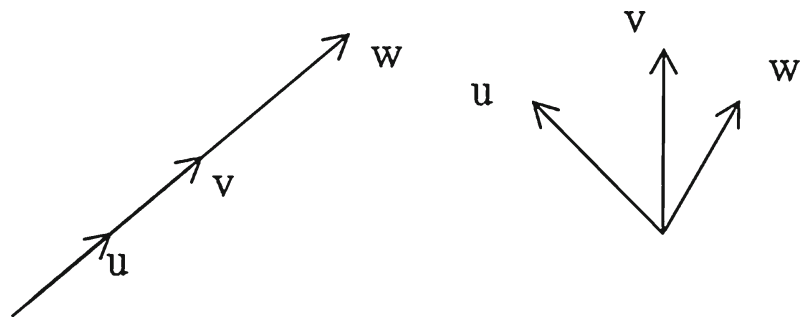

Fig. 6 Linear and radial betweenness of vectors (from Zwarts 1997, p. 81)

are directly relevant for the convexity of prepositional meanings in conceptual spaces (Gärdenfors 2000, p. 172). However, the problem is that continuity is not a general and unified notion, but comes in two different versions that are conceptually related, but not formally. As a consequence, the underlying notion of betweenness and its consequences for the convexity of prepositional regions remains unclear. By analyzing vectors in terms of polar coordinates a notion of polar betweenness and polar convexity becomes available that can unify and strengthen Zwarts' notion of continuity.

There is much more to say about cognitive and computational aspects of spatial representations. Some would argue that it is more natural and efficient to have qualitative instead of quantitative representations, with primitive regions and lines, instead of points (Cohn and Renz 2008). We agree that it would be interesting to see to what 
extent qualitative representations are sufficient to represent the semantics of prepositions, but here we will confine ourselves to some brief remarks. A main thesis of this paper is that most locational and directional prepositions can be represented by regions that are convex, where betweenness is defined in terms of polar coordinates. Thus the notions of betweenness and convexity are central to our analysis. It is true that these notions can be incorporated without assuming a metric, for example in ordered sets (for a survey see Cohn and Renz 2008). However, for many systems of qualitative reasoning, for example the Region Connection Calculus (Cohn et al. 1997) and the Qualitative Trajectory Calculus (van de Weghe et al. 2005), the notion of convexity cannot be expressed. On the other hand there are other systems of qualitative reasoning where convexity is added as an operator $\mathrm{C}$ so that the convex hull of a region $X$ is the region $\mathrm{C}(X)$ (e.g. Randell et al. 1992; Davis 2006). In these systems, the convex regions $X$ can be identified by the fact that they satisfy the identity $\mathrm{C}(X)=X{ }^{4} \mathrm{~A}$ main difference is, however, that the notion of polar convexity is not discussed in any of these systems.

In a sense, our semantics is qualitative since all of our descriptions of the regions of prepositions are based on qualitative criteria for polar coordinates, like their being inside or outside the landmark, near or far, above or below. So in principle, we do not need the metric generated by the polar coordinate system, but only the notion of polar betweenness (with respect to an origo), from which the set of polarly convex sets then can be defined. The important difference from previous analyses is that the set of polarly convex sets is not the same as for Cartesian betweenness.

\section{Semantic Definitions of Locative Prepositions in Polar Coordinates}

We noted earlier that a locative preposition describes a relation between a trajector and a landmark. ${ }^{5}$ This relation can be broken up into two parts (e.g. Jackendoff 1983; Wunderlich 1991). The first part is one of the many different spatial functions that map a landmark to a region, corresponding to different prepositional meanings. The second part is a general function that locates the trajector in that region. In the remainder we will restrict ourselves to the prepositional functions that map landmarks to regions. For example, if $L$ is a landmark, then near $(L)$ will be the region of points that are in proximity to $L$.

We will make the following assumptions and idealizations about how landmarks and regions relate to the spatial domain $S$ that we defined in the previous section. First, we assume that the center of mass of the landmark will function as the origo of $S$ (but see Zwarts and Winter 2000; Regier and Carlson 2001; Mador-Haim and Winter 2015 for much more realistic models). In order to make explicit that a landmark $L$ fixes the origo of the space $S$ in which the preposition is interpreted, we write $S(L)$ for the polar coordinate system defined by landmark $L$. So, if $L_{1}$ and $L_{2}$ are landmarks at different

\footnotetext{
4 In such systems, betweenness can in principle be defined as follows: Point $z$ is between points $x$ and $y$, if and only if, $z$ belongs to all convex regions that both $x$ and $y$ belong to. However, it is unclear whether the betweenness relation defined in this way will have all the appropriate properties (such as satisfying Hilbert's axioms in Grundlagen der Geometrie).

5 Between is an exception since it involves a relation between a trajector and two or more landmarks.
} 
locations in space, then $S\left(L_{1}\right)$ and $S\left(L_{2}\right)$ are different coordinate systems because of their different origos. In $S\left(L_{1}\right)$, the coordinate $\langle r, \theta, \varphi\rangle$ represents a different point than in $S\left(L_{2}\right)$.

Second, we will consider only spherical landmarks, which is of course a strong idealization, but one which for the meanings of most locative prepositions does not result in any major distortions. Clearly, for a full account of spatial semantics, we ultimately need a model that can deal with landmarks of any shape, but we believe that the properties of most prepositions can be studied and modeled more easily with a more restricted type of landmark. ${ }^{6}$ After all, we are not interested in how the shape of a region is determined by the accidental shape of a landmark, but by the lexical properties of a preposition. Moreover, schematizations of different kinds play an important role in spatial language (Herskovits 1998). Third, we assume that the set of points occupied by the landmark is a closed subset of $S(L)$, that is, it contains its boundary.

We now show that by using polar coordinates, locative prepositions can be given a systematic description that also brings out explicitly the spatial features of each preposition. The coordinates of a polar system provide different ways of defining regions in the space $S(L)$ of a landmark. The main distinction is between prepositions that refer to the radius of a point and prepositions that refer to the angles $\theta$ and $\varphi$.

The first distinction based on the radius coordinate is that between internal and external regions, corresponding to the prepositions inside and outside, respectively. Given the idealizations that we have made, we can define these regions as sets of points of which the radius $x$ is either smaller than the radius $\mathrm{r}_{L}$ of the landmark $L$ (inside) or larger (outside), as illustrated in Figs. 7 and 8.

$$
\begin{aligned}
& \operatorname{inside}(L)=\left\{\langle x, \theta, \varphi\rangle \in S(L): x \leq \mathrm{r}_{L}\right\} \\
& \text { outside }(L)=\left\{\langle x, \theta, \varphi\rangle \in S(L): x>\mathrm{r}_{L}\right\}
\end{aligned}
$$

The corresponding regions (or at least their two-dimensional cross-sections) can be diagrammed by shading the area where the points of the regions (that is, the endpoints of the vectors) are.

Without the idealization of a circular landmark, the definitions in (7) would be more complicated, but not crucially different. It would have to refer to the boundary points of a convex landmark $L$ in the following way. If $\langle b, \theta, \varphi\rangle$ is a point on the boundary of $L$, then a point $\langle x, \theta, \varphi\rangle$ is inside $L$ if $x \leq b$ and outside $L$ if $x>b$. If landmarks are not convex, then it becomes much more complicated to determine what is inside and outside (see Ullman 1984, for cognitive issues and Mador-Haim and Winter 2015, for semantic issues).

Next, the space outside the landmark can be divided into points that are near or far, as shown in Figs. 9 and $10 .^{7}$ Of course, at what distance from the landmark the division between 'near' and 'far' is made is dependent on the context, in particular the size of the landmark: what is near the sun in outer space covers a much larger distance

\footnotetext{
6 Along and across are examples of prepositions that are sensitive to the shape of the landmark, requiring axes with respect to which the trajector is located or moving.

7 The space inside the landmark can also be divided in regions that are closer to or further away from its boundary, as shown by combinations like far inside and right inside. We believe that in such cases the boundary is taken as the landmark instead of the origin, a construal which we will not take into consideration.
} 


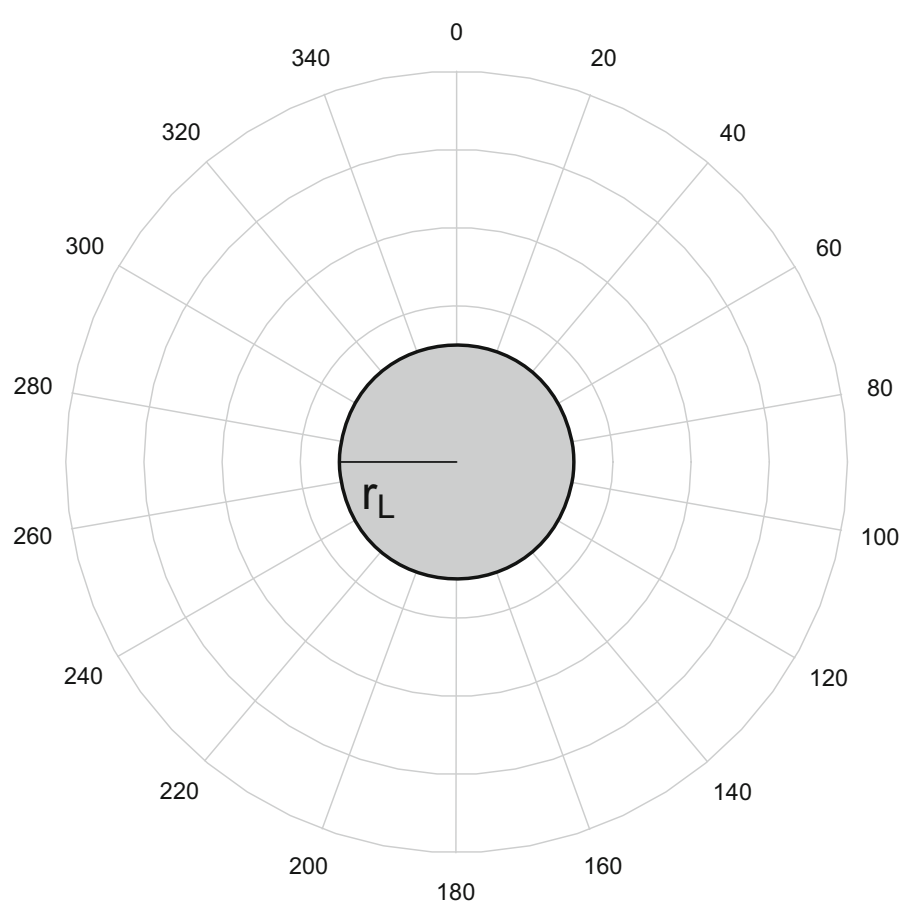

Fig. 7 Inside region

than what is near a golf ball on the ground. Moreover, the division is not sharp but might allow for a vague gap between near and ar. $^{8}$

The definitions of near and far are given in (8) as sets of polar coordinates, for a given landmark with radius $\mathrm{r}_{L}$ and a contextually given norm $\mathrm{c}_{L}$ that represents the demarcation between 'near' and 'far' relative to $L$.

(8) $\operatorname{near}(L)=\left\{\langle x, \theta, \varphi\rangle \in S(L): \mathrm{r}_{L}<x<\mathrm{c}_{L}\right\}$

$\operatorname{far}(L)=\left\{\langle x, \theta, \varphi\rangle \in S(L): \mathrm{c}_{L}<x\right\}$

Then there are prepositions that refer to the angular coordinates. They are based on a frame of reference that defines a region at a particular side of the landmark. There is a rich literature about the different frames of reference, with different terminologies (see Levinson 1996, for an overview). We follow here Levinson, who makes a distinction between absolute frames of reference (based on features of the environment, such as gravitation and compass points), intrinsic frames of reference (based on features of the landmark itself, like its 'active' side), and relative frames of reference (based on the position of an observer looking at the landmark).

\footnotetext{
8 In Gärdenfors (2000, section 3.5), it is shown that there is no conflict between vagueness and the requirement that concepts be represented by convex regions. What convexity requires in relation to vague concepts is that if two object locations $x_{1}$ and $x_{2}$ both satisfy a certain membership criterion, then all objects between $x_{1}$ and $x_{2}$ also satisfy the criterion.
} 


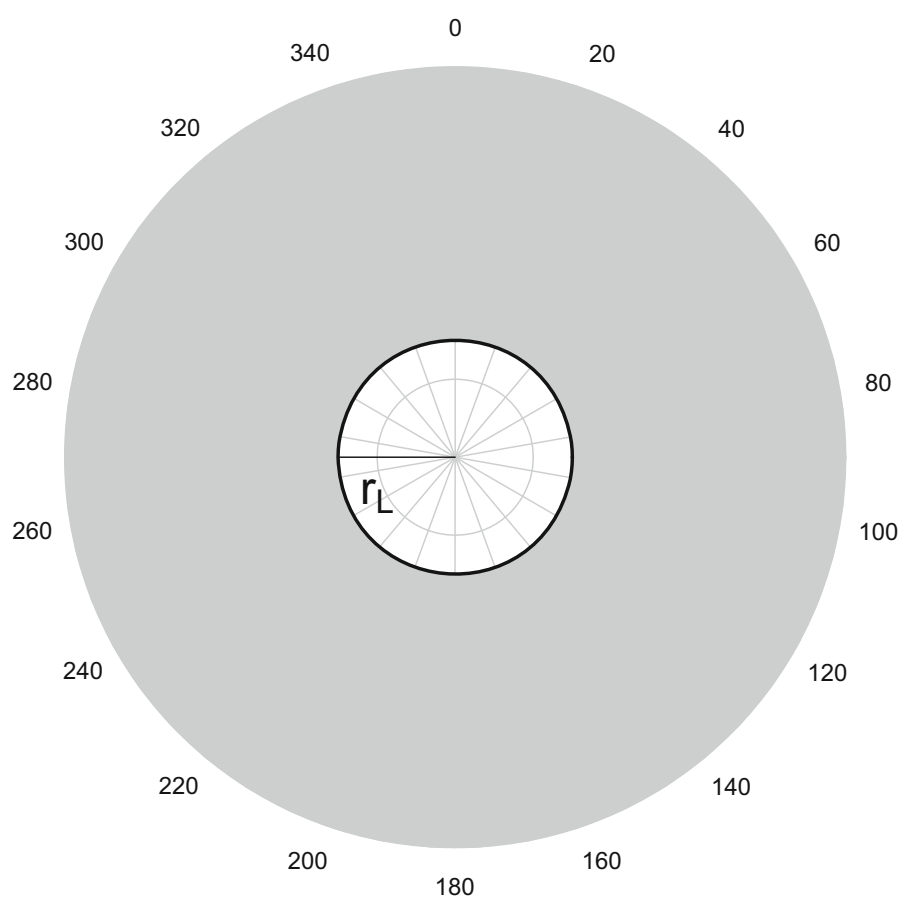

Fig. 8 Outside region

A frame of reference can be represented by a point (a 'unit vector') $\left\langle 1, \theta_{0}, \varphi_{0}\right\rangle$ with particular values for $\theta_{0}$ and $\varphi_{0}$. An absolute frame of reference is a direction that is given independently of any landmark or observer, as a feature of the environment shared by all landmarks under every perspective. For example, this unit vector can be taken to be grav $=\left\langle 1, \theta_{g}, \varphi_{g}\right\rangle$, which is fixed as an absolute frame of reference by the downward direction of gravitation. The prepositions above and below include those points in the region that are close to the unit vector that represents the corresponding vertical axis.

(9) $\operatorname{above}(L)=\left\{\langle x, \theta, \varphi\rangle \in S(L): x>\mathrm{r}_{L}\right.$ and $\langle x, \theta, \varphi\rangle$ is close to -grav $\}$

below $(L)=\left\{\langle x, \theta, \varphi\rangle \in S(L): x>\mathrm{r}_{L}\right.$ and $\langle x, \theta, \varphi\rangle$ is close to grav $\}$

Two points $a$ and $b$ are close if there is a degree $d$ such that $\angle a b<d$. The closeness in (9) is a matter of context and vagueness, but for the sake of concreteness we will simply assume that $d=45^{\circ}$. This yields the infinite cones that we see in Figs. 11 and 12 (showing two-dimensional cross-sections of regions that are actually threedimensional).

An intrinsic frame of reference is a way of assigning directions that depends on features of the landmark, like where its face and senses are (for humans and animals) or where its interactive side is (for animals). For a landmark $L$ at a particular moment

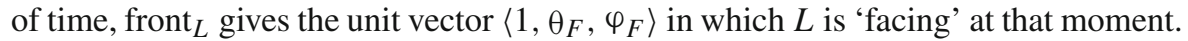
In its canonical orientation, front ${ }_{L}$ will be horizontal, i.e. orthogonal to the absolute direction given by the gravitational vector. Similarly, we can assume a function right $L$ 


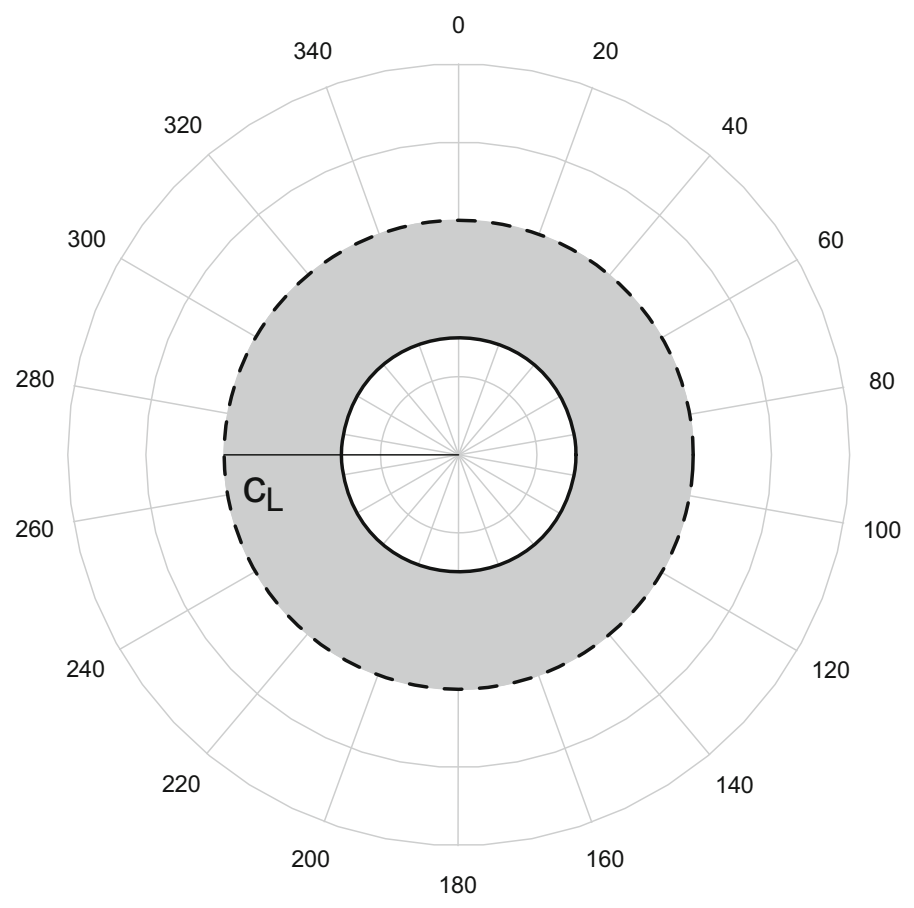

Fig. 9 Near region

that assigns a direction orthogonal to front ${ }_{L}$. Notice that these intrinsic frames of reference are functions of the landmark $L$. The intrinsic meaning of in front of, behind, right of, and left of can then be defined in (10).

(10) in-front-of int $(L)=\left\{\langle x, \theta, \varphi\rangle \in S(L): x>\mathrm{r}_{L}\right.$ and $\langle x, \theta, \varphi\rangle$ is close to front $\left._{L}\right\}$ behind $_{\text {int }}(L)=\left\{\langle x, \theta, \varphi\rangle \in S(L): x>\mathrm{r}_{L}\right.$ and $\langle x, \theta, \varphi\rangle$ is close to - front $\left._{L}\right\}$ right-of $_{\text {int }}(L)=\left\{\langle x, \theta, \varphi\rangle \in S(L): x>\mathrm{r}_{L}\right.$ and $\langle x, \theta, \varphi\rangle$ is close to right $\left._{L}\right\}$ left-of int $(L)=\left\{\langle x, \theta, \varphi\rangle \in S(L): x>\mathrm{r}_{L}\right.$ and $\langle x, \theta, \varphi\rangle$ is close to - right $\left._{L}\right\}$

The corresponding regions are cone-shaped again, like those of above and below.

Another preposition that can be based on an intrinsic frame of reference is beside. Following Leech (1974) and Svenonius (2008) we analyze beside here as involving proximity to the sides of the landmark. It seems reasonable to assume that the landmark has a left and right side and that the definition of beside (and next to, its synonym for our purposes) should be as follows: ${ }^{9}$

\footnotetext{
9 An anonymous reviewer wondered whether it would not be possible to have only a region at one side in a particular context of use. This would essentially move the disjunction one level up: beside $(L)=$ $\left\{\langle x, \theta, \varphi\rangle \in S(L): \mathrm{r}_{L}\left\langle x \leq \mathrm{c}_{L}\right.\right.$ and $\langle x, \theta, \varphi\rangle$ is close to $\left.\operatorname{right}_{L}\right\}$ OR beside $(L)=\{\langle x, \theta, \varphi\rangle \in S(L)$ : $\mathrm{r}_{L}<x \leq \mathrm{c}_{L}$ and $\langle x, \theta, \varphi\rangle$ is close to -right $\left.{ }_{L}\right\}$. Although this is a potential solution for the non-p-convexity of beside noted in Sect. 5, it is a departure from the way the region of beside has been treated in earlier literature. Moreover, it is not immediately clear how this approach would deal with complex or multiplex trajectors that are located on both sides simultaneously, like a couple sitting next to the Queen or grass growing beside the road. This is where an independent dimension of distributivity comes in De Vries (2015). How trajectors 'distribute' over regions and what this implies for those regions goes beyond this article.
} 


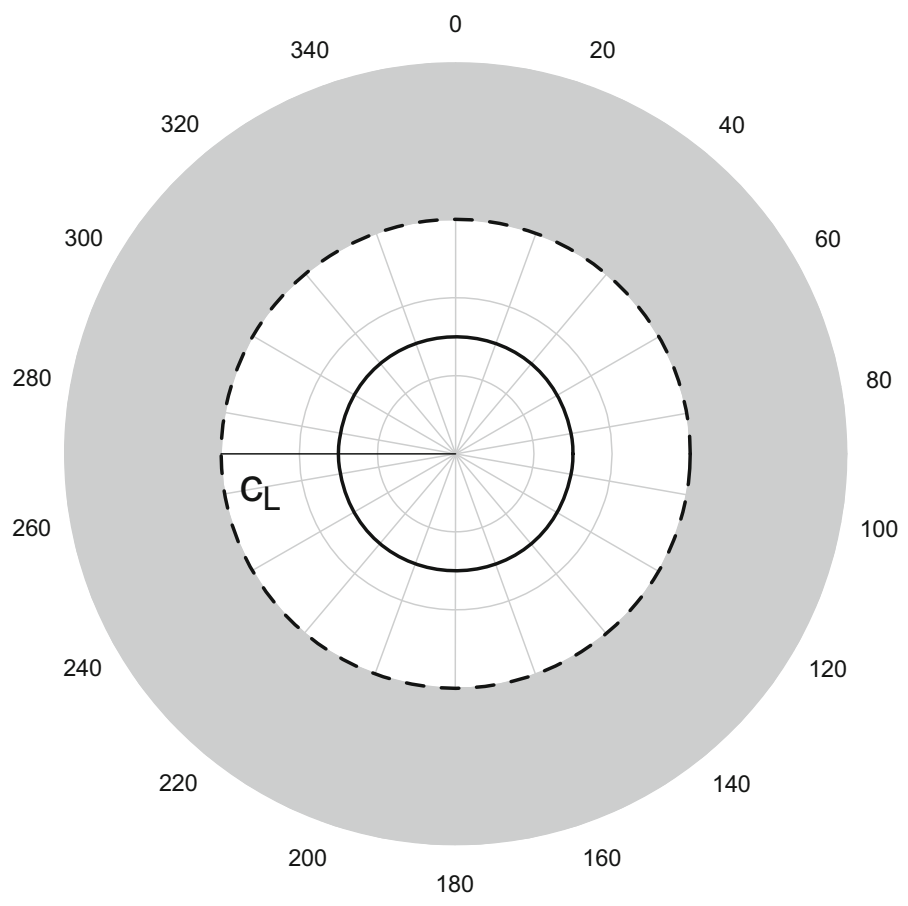

Fig. 10 Far region

(11) beside $(L)=\left\{\langle x, \theta, \varphi\rangle \in S(L): \mathrm{r}_{L}<x \leq \mathrm{c}_{L}\right.$ and $\langle x, \theta, \varphi\rangle$ is close to right $_{L}$ or to - right $\left._{L}\right\}$

What makes this region different from the regions of the (other) axis-based prepositions is that it stretches out in two opposite directions, as shown in Fig. 13.

The prepositions in front of, behind, right, and left can also be interpreted with respect to a relative frame, which can be represented by making the front and right sides of $L$ dependent on an observer $O$. Front $L, O$ gives the side of $L$ that is oriented towards $O$ and right $_{L, O}$ gives the side of $L$ that has the same orientation as $O$.

(12) in-front-of $\mathrm{rel}_{\mathrm{rel}}(L)=\left\{\langle x, \theta, \varphi\rangle \in S(L): x>\mathrm{r}_{L}\right.$ and $\langle x, \theta, \varphi\rangle$ is close to front $\left.L, O\right\}$ behind $_{\text {rel }}(L)=\left\{\langle x, \theta, \varphi\rangle \in S(L): x>\mathrm{r}_{L}\right.$ and $\langle x, \theta, \varphi\rangle$ is close to - front $\left._{L, O}\right\}$ right-of $_{\text {rel }}(L)=\left\{\langle x, \theta, \varphi\rangle \in S(L): x>\mathrm{r}_{L}\right.$ and $\langle x, \theta, \varphi\rangle$ is close to right r,$\left.O\right\}$ left-of $_{\text {rel }}(L)=\left\{\langle x, \theta, \varphi\rangle \in S(L): x>\mathrm{r}_{L}\right.$ and $\langle x, \theta, \varphi\rangle$ is close to - right $\left._{L, O}\right\}$

We turn to the preposition between, which is, in its most prominent use, based on two landmarks, as in the prepositional phrase between John and Mary (Habel 1989; van der Zee and Watson 2004). There are two origos for the polar coordinates defined by this PP, John and Mary, and therefore two polar coordinate systems, $S$ (John) and $S$ (Mary). Consider a point between John and Mary. This point corresponds to coordinates $\langle x, \theta, \varphi\rangle \in S(\mathrm{John})$ such that $\langle x, \theta, \varphi\rangle$ is directed towards Mary; it also corresponds to coordinates $\left\langle x^{\prime}, \theta^{\prime}, \varphi^{\prime}\right\rangle \in S$ (Mary) such that $\left\langle x^{\prime}, \theta^{\prime}, \varphi^{\prime}\right\rangle$ is directed towards John. We can say that $\langle x, \theta, \varphi\rangle$ and $\left\langle x^{\prime}, \theta^{\prime}, \varphi^{\prime}\right\rangle$ describe the same point in 


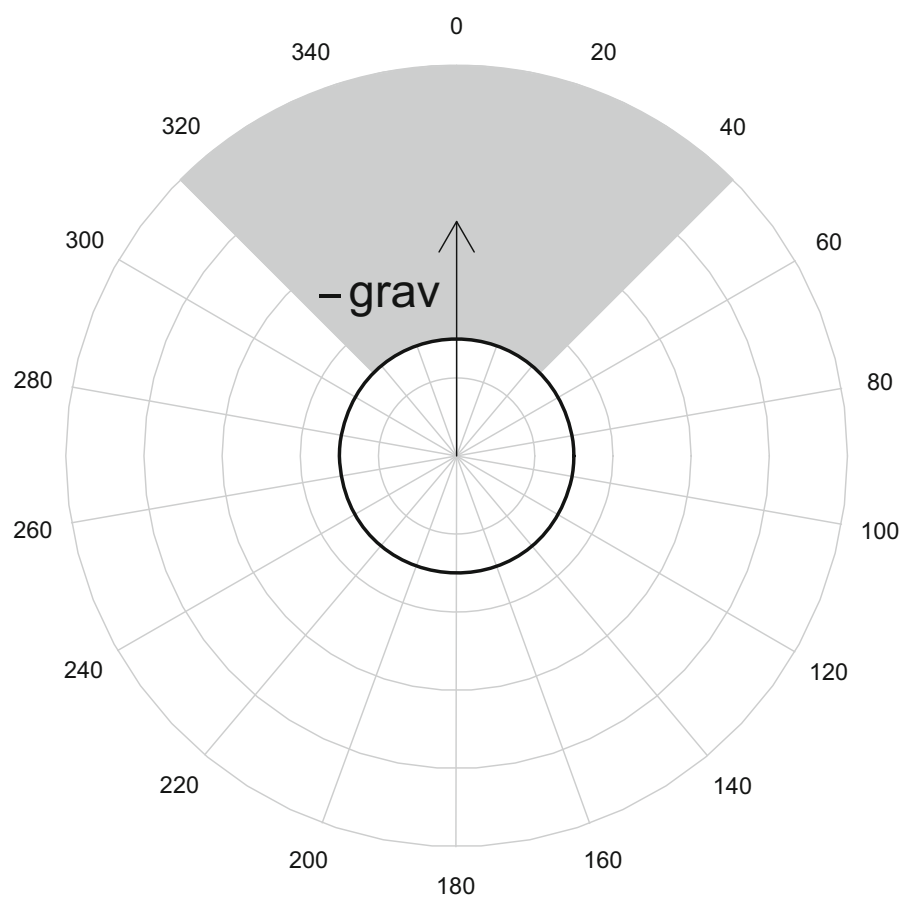

Fig. 11 Above region

space, but from different origos. This leads us to the following quite rudimentary definition of between, where $\operatorname{dir}\left(L, L^{\prime}\right)$ is the vector in $S(L)$ that represents the point of $L^{\prime}$ closest to $L$, as defined in (13), and $\langle x, \theta, \varphi\rangle \approx\left\langle x^{\prime}, \theta^{\prime}, \varphi^{\prime}\right\rangle$ represents that those two coordinate triples represent the same point in space, even though they are defined with respect to different origos.

(13) $\operatorname{dir}\left(L, L^{\prime}\right)=$ the shortest vector $\langle x, \theta, \varphi\rangle \in$ outside $(L)$ for which there is a $\left\langle x^{\prime}, \theta^{\prime}, \varphi^{\prime}\right\rangle \in \operatorname{inside}\left(L^{\prime}\right)$ such that $\langle x, \theta, \varphi\rangle \approx\left\langle x^{\prime}, \theta^{\prime}, \varphi^{\prime}\right\rangle$

(14) between-and $\left(L, L^{\prime}\right)=\left\{\langle x, \theta, \varphi\rangle \in S(L): \operatorname{dir}\left(L, L^{\prime}\right)=\langle d, \theta, \varphi\rangle\right.$ and $\left.x<d\right\}$

In this case the region between-and $\left(L, L^{\prime}\right)$ consists of the points lying on the shortest line segment connecting the two landmarks $L$ and $L^{\prime}$, as illustrated in Fig. 14.

Note that between $\left(L, L^{\prime}\right)$ and between $\left(L^{\prime}, L\right)$ correspond to the same line, but with different origos. Obviously, this is a very narrow definition, which needs to be elaborated, but it captures the essential nature of between. One way to extend the definition is to allow a wider (vague) region between the landmarks. Another extension is that between can refer to multiple landmarks as in the expression between the trees. In the latter case, the convex hull of the landmarks would be a plausible representation of the meaning (presuming a unique origo in relation to which polar convexity is defined).

In summary, the space around a landmark can be represented in terms of polar coordinates and a locative preposition carves out a region in this space by imposing 


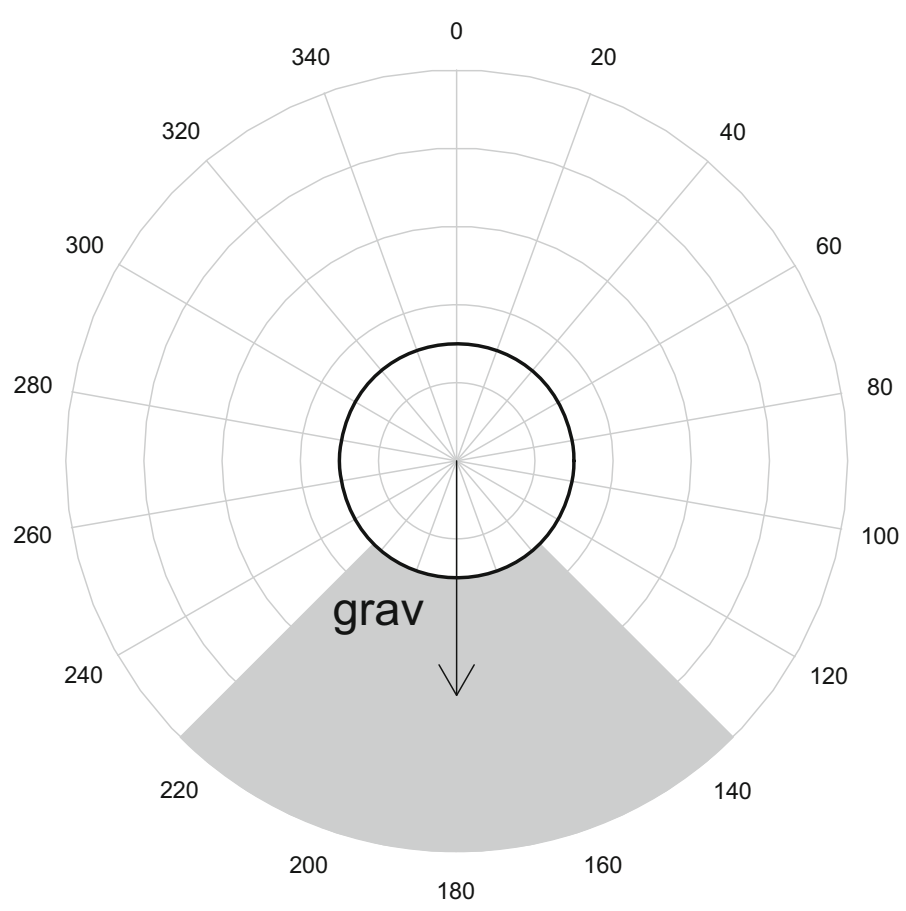

Fig. 12 Below region

simple conditions on the values of the polar coordinates. We will now consider whether the regions defined in this way are also polarly convex.

\section{The Convexity of Locative Prepositions}

Given the notions of polar betweenness and polar convexity that we defined in Sect. 3, the question is now whether the regions of locative prepositions that we defined in Sect. 4 are convex in this sense.

The preposition inside is trivially p-convex, because of the idealizing assumption about landmarks we have made. The region corresponding to inside is always the interior of a circle, which is p-convex (as well as convex in the Cartesian sense, of course). In reality, inside can be used with landmarks of any shape, like inside the cathedral, with the cathedral having the shape of a cross, and the resulting interior region is not convex or p-convex in that case. We are interested in the general properties that prepositional categories have, independently of the more accidental properties of the landmarks that they are based on. Inside is not relevant for studying such properties, because the shape of its region is completely determined by the landmark that it applies to. The 'exterior' prepositions are where p-convexity has to be put to the test.

Consider therefore outside, near, and far, that are problematic for the ordinary, Cartesian notion of convexity, because there is a gap in the center of the region. If position $a$ to the east of my house is outside (near, far) and a position $b$ to the west 


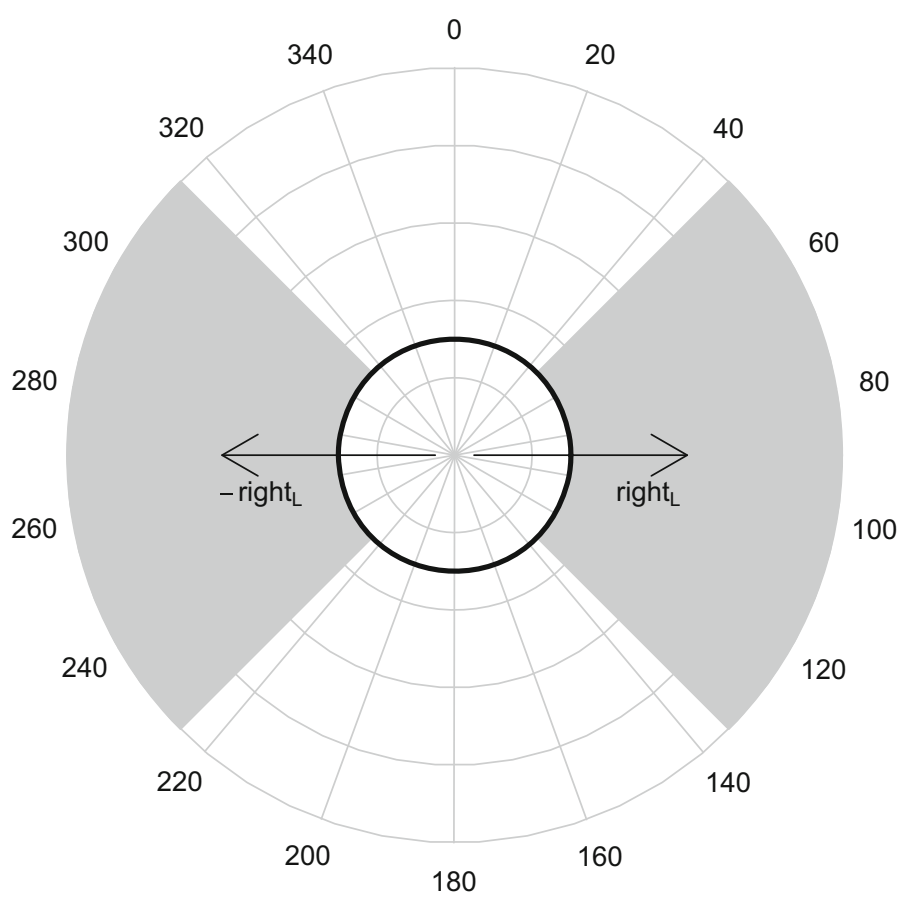

Fig. 13 Beside region

Fig. 14 The region between two landmarks $L$ and $L^{\prime}$

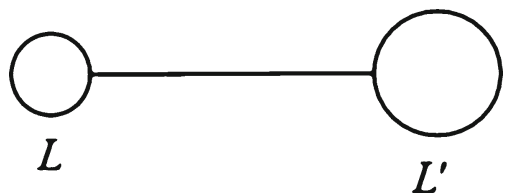

is outside (near, far), then there are definitely positions between $a$ and $b$ that are not outside (near, far). But this description depends on Cartesian betweenness. If we apply p-betweenness to these regions, then they are p-convex in terms of polar coordinates. As we saw in Sect. 3, the exterior of a sphere (the shape of outside and far regions) is p-convex and also the region between two spheres (the shape of near regions). Crucially, in these cases the curved nature of the p-convexity line between the two points $a$ and $b$ leads around the gap in the middle. Hence, a point p-between $a$ to the east and $b$ to the west is a point either to the north or to the south (unless $a$ and $b$ are on diametrically opposite sides, because then p-betweenness is not defined, see Sect. 3).

The regions defined by the 'angular' prepositions above, below, in front, behind, left, and right are all p-convex according to the definitions of Sect. 4, because they are the intersection of two basic p-convex regions: the exterior of a sphere (because of the condition that $x>\mathrm{r}_{L}$ ) and an infinite cone (because of the condition that the angular coordinates are close to the unit vector that represents the axis). What frame of reference underlies the preposition does not affect the p-convexity. What is important for the p-convexity of these regions is that they are located at one side of the landmark 
only. Regions defined by between are p-convex because between is taken very strictly, resulting in a line segment between the two landmarks. We are left then with the pconvexity of the preposition beside, for which we defined a region that consists of two parts on opposite sides of the landmark. Given definition (11) this region is not p-convex (see Fig. 13). However, we do not want to conclude that beside falsifies pconvexity, until we have explored alternative ways of defining its meaning. As Leech (1974) points out for the synonym at the side of:

In a very general sense, at the side of has no reference to vertical or horizontal orientation: in an astronomical context, Star $x$ is at the side of star $y$ may mean no more than that the two stars are in spatial proximity. A less general sense of at the side of is found in I placed my hat at the side of his, which means '... close to his on a horizontal plane'; there is a sense, that is, in which ' $x$ is at the side of $y$ ' excludes the possibility of vertical proximity. A third and still more specific sense is observed in His car was at the side of mine, where at the side of is in contrast to in front of and behind, and therefore limits proximity to the secondary horizontal axis. (Leech 1974, p. 169)

If we follow this view, beside does have a meaning that is p-convex, namely a meaning of spatial proximity (like near). We propose that that is the basic meaning of beside and that the more restricted uses result from the pragmatic competition with abovelbelow (excluding vertical proximity) and in front oflbehind (excluding proximity to those sides). It has the same meaning as near, but it forms a lexical field with projective prepositions like above and behind, which restrict its meaning in contextually dependent ways. In other words, there is an element of negation in beside: "near, but not above, behind, etc.', somewhat similar to an implicature. If this is true, then it teaches us an important lesson about convexity in general. Cases of non-convexity might actually result from the pragmatic interaction between a convex category and another category that partially overlaps it, creating an 'indentation' that causes non-convexity.

When we turn to complex prepositions, run-of-the-mill cases satisfy convexity (given our assumptions about landmarks):

(15) a. An exit sign is located 1 feet above the exit.

b. The hotel is too far outside the city.

Only artificial cases such as (16a) and (16b) will violate p-convexity:

(16) a. Click a position diagonally above the circle.

b. The current level is thus exactly one or exactly two meters above the official level.

In other words, not only basic prepositions are p-convex, but even many of the complex ones. This means that p-convexity gains additional support as a general semantic constraint for locative prepositions, as well as the underlying use of polar coordinates. Of course, the principle of p-convexity should also be analyzed cross-linguistically, against a much wider range of languages and locative adpositions, but that is beyond the scope of this paper. 


\section{Directional Prepositions with Polar Coordinates}

\subsection{Directional Prepositions and Paths}

We now turn from locative prepositions to directional prepositions, which are used to describe how a trajector is moving with respect to a landmark:

(17) a. The fox went into the forest.

b. The dog came off the table.

c. The fox jumped through the hedge.

The previous section introduced the formalism for representing the location of a trajector in terms of one vector. If a trajector is moving or if it is extended in shape, then we need the notion of a path, i.e. a directed curve (see for instance Jackendoff 1983; Talmy 2000; Eschenbach et al. 2000; Zwarts 2005; Kracht 2008, and many others). There are different ways to represent a path, but we will represent it here as a function from the real interval $[0,1]$ to $S$, continuous in the standard calculus sense. The values of the interval $[0,1]$ do not represent moments of time, but they are an ordering mechanism. We also assume that a path has a constant 'velocity', i.e. that its first derivative is constant (see Zwarts 2005; Kracht 2008, for more details). Note that instead of [0,1] we could also have taken a discrete domain for the function, because a path might not always need or allow for a continuous curve, but may be just a finite sequence of positions, or, minimally, only two positions, a starting point and an endpoint. What is important is that the path represents 'locations in sequence' so to say. In the sequel we also make the assumption that the path is simple, that is, it does not cross itself. This can be defined by saying that for all $i, j \in[0,1]$, such that $i \neq j, p(i) \neq p(j)$.

We can refer to the starting point of a path $p$ as $p(0)$, the end point is $p(1)$ and for any $i \in(0,1), p(i)$ is an intermediate point. All the points of a path are represented in terms of polar coordinates taken from $S$. It will be convenient later on to refer to these coordinates in the following ways:

(18) If $p(i)=\langle x, \theta, \varphi\rangle$, then $\operatorname{radius}(p(i))=x, \operatorname{azimuth}(p(i))=\theta$, and $\operatorname{polar}(p(i))=\varphi$.

In Fig. 15 a path $p$ with respect to a landmark $L$ is shown with $p(0)=a$ and $p(1)=b$, with radius $(p(0))>\mathrm{r}_{L}$ and $\operatorname{radius}(p(1))<\mathrm{r}_{L}$. If the situation holds in the $x y$-plane, then for every $i \in[0,1]$, polar $(p(i))=90^{\circ}$.

\subsection{Defining the Meaning of Directional Prepositions}

Among the directional prepositions one usually distinguishes the following three types (e.g. Jackendoff 1983; Kracht 2002; Pantcheva 2010):

(19) goal prepositions: to, into, onto, towards

source prepositions: from, out of, off, away from

route prepositions: through, over, along, around, across

In addition, it is possible to get directional readings for locative prepositions: 


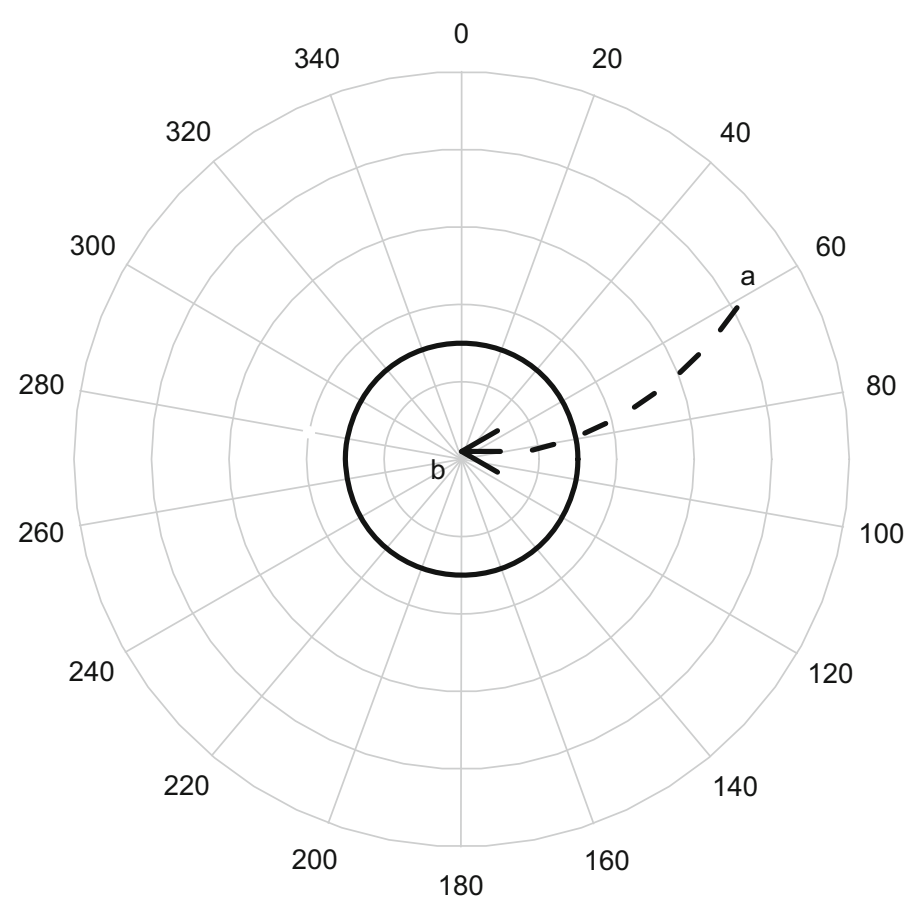

Fig. 15 A path into L

(20) goal: (to go) below, behind, ... something

source: (to come) from below, from behind, ... something

route: (to pass) below, behind, ... something

The source needs to be marked by the source preposition from, while the possibility to get a goal or route interpretation depends very much on the verb and other factors (see for example Gehrke 2008; Nikitina 2008).

Most of the directional prepositions can be represented as imposing a locative condition on a particular part of the path, for instance on the end point (goal) or starting point (source). Stricter and weaker definitions are possible, as discussed in Zwarts (2005) and other works, but here we will use definitions that only involve opposite conditions on the starting point and end point. Suppose that $P(S(L))$ represents the set of paths in the space $S(L)$ of a landmark $L$. (21) gives examples of definitions of goal prepositions, as functions from landmarks to sets of paths, and (22) does the same for source prepositions. ${ }^{10}$

(21) Goal prepositions

to $(L)=\{p \in P(S(L)): p(1) \in \operatorname{near}(L)$ and $p(0) \in \operatorname{far}(L)\}$

$\operatorname{into}(L)=\{p \in P(S(L)): p(1) \in \operatorname{inside}(L)$ and $p(0) \in \operatorname{outside}(L)\}$

to-behind $(L)=\{p \in P(S(L)): p(1) \in \operatorname{behind}(L)$ and $p(0) \notin$ behind $(L)\}$

\footnotetext{
10 The strict definition would require that there is a $k \in(0,1)$ such that for all $i<k, p(i) \in \operatorname{near}(L)$ and for all $i \geq k, p(i) \in \operatorname{far}(L)$, enforcing a transition from one region to another.
} 


\section{(22) Source prepositions}

from $(L)=\{p \in P(S(L)): p(0) \in \operatorname{near}(L)$ and $p(1) \in \operatorname{far}(L)\}$ out-of $(L)=\{p \in P(S(L)): p(0) \in \operatorname{inside}(L)$ and $p(1) \in$ outside $(L)\}$ from-behind $(L)=\{p \in P(S(L)): p(0) \in \operatorname{behind}(L)$ and $p(1) \notin$ behind $(L)\}$

Notice that goal and source prepositions differ in whether they apply the locative condition to the end point or the starting point, respectively. A way to handle certain route prepositions is to view them as sequences of two conjoined paths: ${ }^{11}$ by or past a landmark means that the first path goes to the landmark and the second path goes away from the landmark; through a landmark means that the first path goes into the landmark and the second path goes out of from the landmark; and going behind a landmark means that the first path goes to behind the landmark and the second path goes from behind the landmark.

\section{(23) Route prepositions}

$\operatorname{by}(L)=\operatorname{past}(L)=\{p \in P(S(L))$ : there is a path $q \in \operatorname{to}(L)$ and a path $r \in \operatorname{from}(L)$ and $\left.p=q^{\wedge} r\right\}$

$\operatorname{through}(L)=\{p \in P(S(L))$ : there is a path $q \in \operatorname{into}(L)$ and a path $r \in$ out-of $(L)$ and $p=q \wedge r\}$

via-behind $(L)=\{p \in P(S(L))$ : there is a path $q \in$ to-behind $(L)$ and a path $r \in$ from-behind $(L)$ and $\left.p=q^{\wedge} r\right\}$

The prepositions towards and away from involve a comparison of the radius (the distance to the landmark) at the beginning and end of the path:

(24) towards $(L)=\{p \in P(S(L)): \operatorname{radius}(p(1))<\operatorname{radius}(p(0))$ and for all $\left.i \in[0,1] \operatorname{radius}(p(i))>\mathrm{r}_{L}\right\}$

away-from $(L)=\{p \in P(S(L)): \operatorname{radius}(p(0))<\operatorname{radius}(p(1))$ and for all $\left.i \in[0,1] \operatorname{radius}(p(i))>\mathrm{r}_{L}\right\}$

We now come to a class of directional prepositions that are at a level of greater complexity than the others, namely around, across, and along. What makes them different from the other route prepositions is that they are not specified in terms of a location at a particular point of the path, but in terms of the shape or orientation of the path as a whole. Around paths are round in some sense, across paths are orthogonal to the main axis of the landmark, along paths parallel (see, for instance Landau and Jackendoff 1993 for more discussion).

We have to set aside across and along, because, as we already mentioned, they involve landmarks that are elongated, which goes beyond our simple model of the landmark as a region around an origo. This leaves us with around, which is a preposition with a wide range of meanings (Zwarts 2003). A very strict interpretation of around (restricted to one plane, with $\varphi=90^{\circ}$ ) takes it as corresponding to a full and perfect circular path:

(25) $\operatorname{around}_{\text {circle }}(L)=\left\{p \in P(S(L))\right.$ : there is an $x>\mathrm{r}_{L}$ and an $\alpha \in\left[0^{\circ}, 360^{\circ}\right)$, such that for all $i \in[0,1] p(i)=\left\langle x,\left(\alpha+i \cdot 360^{\circ}\right), 90^{\circ}\right\rangle$ or for all $i \in[0,1] p(i)=$ $\left.\left\langle x,\left(\alpha-i \cdot 360^{\circ}\right), 90^{\circ}\right\rangle\right\}$

\footnotetext{
${ }^{11}$ See Zwarts (2005) for a definition of such an operation on paths represented here with the symbol ${ }^{\wedge}$.
} 
$x$ corresponds to the radius of the circular path and $\alpha$ to the value of the azimuth angle at which the path starts. The disjunction allows the path to go clockwise and counterclockwise. We can extend this meaning by allowing smaller parts of the circumference to be covered, using a factor $k$. If $k=0.5$, then the path corresponds to a semicircle, if $k=0.25$ to a quarter circle. The value of $k$ should be sufficiently large for the path to be circular.

(26) $\operatorname{around}_{\text {partcircle }}(L)=\left\{p \in P(S(L))\right.$ : there is an $x>\mathrm{r}_{L}$, an $\alpha \in\left[0^{\circ}, 360^{\circ}\right)$, and a sufficiently large $k \in(0,1)$ such that for all $i \in[0,1] p(i)=$ $\left\langle x, \alpha+k \cdot i \cdot 360^{\circ}, 90^{\circ}\right\rangle$ or for all $\left.i \in[0,1] p(i)=\left\langle x, \alpha-k \cdot i \cdot 360^{\circ}, 90^{\circ}\right\rangle\right\}$

Another sense of around is a path that encloses the landmark completely on all sides (in the $x y$ plane), as defined in (27).

(27) $\operatorname{around}_{\text {enclosure }}(L)=\left\{p \in P(S(L))\right.$ : for every $\theta \in\left[0^{\circ}, 360^{\circ}\right)$ there is an $i \in[0,1]$ and an $x>\mathrm{r}_{L}$ such that $\left.p(i)=\left\langle x, \theta, 90^{\circ}\right\rangle\right\}$

This definition includes paths that spiral around the landmark, for instance. There are more possibilities (see Zwarts 2003), but this gives an idea of the variety that some route prepositions exhibit.

\subsection{Convexity of Directional Prepositions}

If we focus on simple paths that are defined as mappings from the interval $[0,1]$ to points $\langle x, \theta\rangle$ in one plane (omitting the polar coordinate $\varphi$ ), the following general definition adopts the polar geometry for paths: ${ }^{12}$

(28) Let $p_{a}(i)$ and $p_{c}(i)$, where $i \in[0,1]$, be two paths mapping onto $\left\langle x_{a}(i), \theta_{a}(i)\right\rangle$ and $\left\langle x_{c}(i), \theta_{c}(i)\right\rangle$ respectively. Then the path $p_{b}(i)$ is said to be polarly between (p-between) $p_{a}(i)$ and $p_{c}(i)$, if and only if, there is some $k, 0<k<1$, such that for all $i \in[0,1], p_{b}(i)=\left\langle k x_{a}(i)+(1-k) x_{c}(i), k \theta_{a}(i)+(1-k) \theta_{c}(i)\right\rangle$ iff

12 In most cases, a more general definition will work, which allows the value of $k$ to vary over the interval [0,1]: for all $i \in[0,1]$, there is some $k, 0<k<1$, such that $p_{b}(i)=\left\langle k r_{a}(i)+(1-k) r_{c}(i), k \theta_{\mathrm{a}}(i)+(1-\right.$ $\left.k) \theta_{c}(i)\right\rangle$ iff $\left|\theta_{a}(i)-\theta_{c}(i)\right|<180^{\circ}$ and $p_{b}(i)=\left\langle k x_{a}(i)+(1-k) x_{c}(i), k \theta_{a}(i)+(1-k)\left(\theta_{c}(i)-360^{\circ}\right)\right\rangle$ iff $\left|\theta_{a}(i)-\theta_{c}(i)\right|>180^{\circ}$. However, there are examples that lead to counterintuitive results. Consider the preposition around $_{\text {partcirc }}$, for which we assumed its path should cover at least a quarter of the circumference of the landmark. Take two paths that fall under this definition, both with a constant radius of 1 :

$p_{1}$ : describes a path from $0^{\circ}$ to $90^{\circ}$ clockwise

$p_{2}$ : describes a path from $90^{\circ}$ to $180^{\circ}$ clockwise

According to this more general definition, the following path $p_{3}$ (also with radius 1) is p-between $p_{1}$ and $p_{2}$ :

$\mathrm{p}_{3}$ : describes a path from $89^{\circ}$ to $91^{\circ}$ clockwise

The reason: for all $i, p_{3}(i)$ is between $p_{1}(i)$ and $p_{2}(i)$. For instance, $p_{3}(0)=89^{\circ}$ is between $0^{\circ}$ and $90^{\circ}$, $p_{3}(0.5)=90^{\circ}$ is between $45^{\circ}$ and $135^{\circ}, p_{3}(1)=91^{\circ}$ is between $90^{\circ}$ and $180^{\circ}$. The problem, however, is that a path over 2 degrees would not be called an around path, because it does not cover enough of the circumference of the landmark. In other words, that property of around is lost under this more general notion of p-betweenness. 


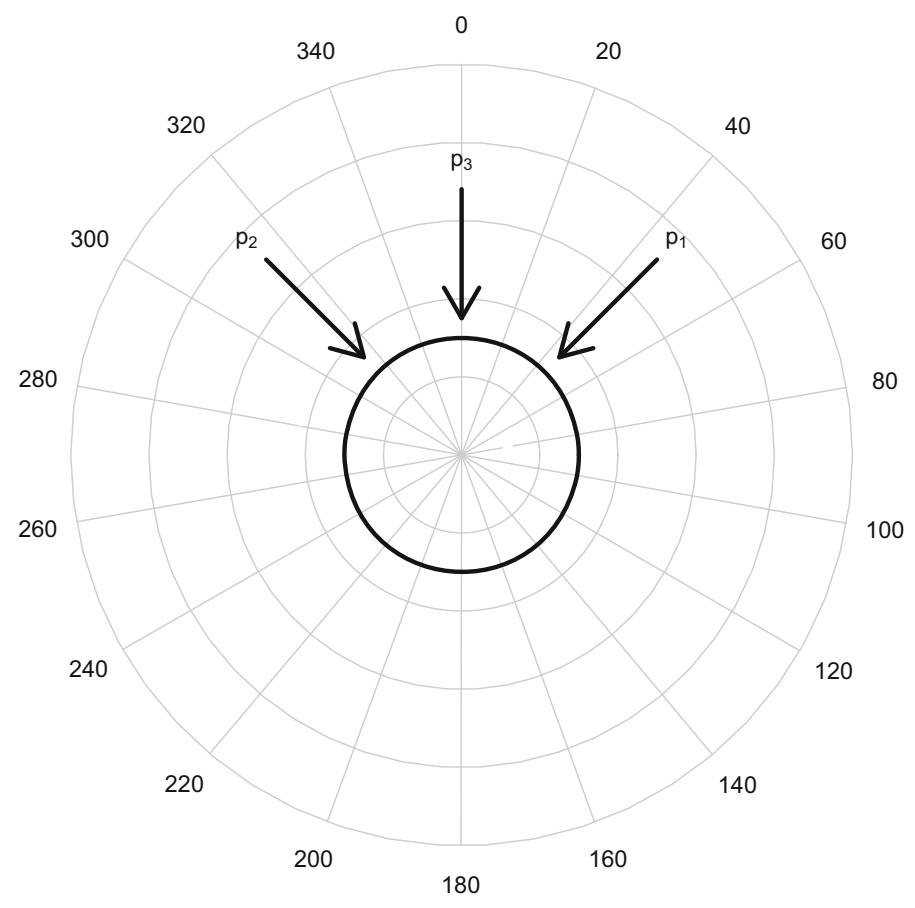

Fig. 16 Path $p_{3}$ is p-between $p_{1}$ and $p_{2}$

$$
\begin{aligned}
& \left|\theta_{a}(i)-\theta_{c}(i)\right|<180^{\circ} \text { and } p_{b}(i)=\left\langle k x_{a}(i)+(1-k) x_{c}(i), k \theta_{a}(i)+(1-k)\right. \\
& \left.\left(\theta_{c}(i)-360^{\circ}\right)\right\rangle \text { iff }\left|\theta_{a}(i)-\theta_{c}(i)\right|>180^{\circ} .13
\end{aligned}
$$

Given this definition it is easy to prove that the meanings of the goal prepositions above are polarly convex. Convexity for into follows from convexity of inside together with the convexity of outside. More precisely, if $p_{1}$ and $p_{2}$ are two paths such that $p_{1}(1) \in \operatorname{inside}(L)$ and $p_{2}(1) \in \operatorname{inside}(L)$ for any $L$, then for any path $p_{3}$ between $p_{1}$ and $p_{2}$, it will also hold that $p_{3}(1) \in \operatorname{inside}(L)$; and if $p_{1}(0) \in \operatorname{outside}(L)$ and $p_{2}(0) \in \operatorname{outside}(L)$ for any $L$, then for any path $p_{3}$ between $p_{1}$ and $p_{2}$, it will also hold that $p_{3}(0) \in$ outside $(L)$. A parallel proof can be given for out of.

Similarly, the convexity of to follows from the fact that the regions for near and far are convex. To be precise, if $p_{1}$ and $p_{2}$ are two paths such that $p_{1}(1) \in \operatorname{near}(L)$ and $p_{2}(1) \in \operatorname{near}(L)$ for any $L$, then for any path $p_{3}$ between $p_{1}$ and $p_{2}$, it will also hold that $p_{3}(1) \in \operatorname{near}(L)$; and if $p_{1}(0) \in \operatorname{far}(L)$ and $p_{2}(0) \in \operatorname{far}(L)$ for any $L$, then for any path $p_{3}$ between $p_{1}$ and $p_{2}$, it will also hold that $p_{3}(0) \in \operatorname{far}(L)$. See Fig. 16 for a graphical illustration. An analogous argument holds for from, and similar arguments can be made for (to) behind and from behind, since the regions for behind and its complement outside the landmark are convex.

\footnotetext{
13 As in definition (5) this can be applied also for the case when $a$ (or $c$ ) is the origo by putting $\theta_{a}=\theta_{c}$ and $\varphi_{a}=\varphi_{c}$.
} 
The polar convexity of towards $(L)$ is easy to prove: if $p_{1}(i)$ and $p_{2}(i)$ are two paths $\operatorname{such}$ that $\operatorname{radius}\left(p_{1}(1)\right)<\operatorname{radius}\left(p_{1}(0)\right)$ and for all $i \in[0,1] \operatorname{radius}\left(p_{1}(i)\right)>\mathrm{r}_{L}$, and $\operatorname{radius}\left(p_{2}(1)\right)<\operatorname{radius}\left(p_{2}(0)\right)$ and for all $i \in[0,1] \operatorname{radius}\left(\mathrm{p}_{2}(i)\right)>\mathrm{r}_{L}$, then for any path $p_{3}$ p-between $p_{1}$ and $p_{2}$, it will also hold that radius $\left(p_{3}(1)\right)<\operatorname{radius}\left(p_{3}(0)\right)$ and for all $i \in[0,1] \operatorname{radius}\left(p_{3}(i)\right)>\mathrm{r}_{L}$. An analogous argument shows that away from is convex.

Given the definitions in (23), the convexity of the route prepositions follows essentially from the convexity of the set of points that conjoin the two paths, in analogy with the convexity for the goal prepositions. A point conjoining two paths $p$ and $q$ is a point for which $p(1)=q(0)$. It is interesting to note that with our definition of betweenness for paths, conjoining paths preserves convexity: If $P$ and $Q$ are convex sets of paths and $P^{\wedge} Q$ is the set of conjoinings of paths from $P$ and $Q$, then $P^{\wedge} Q$ is convex too. ${ }^{14}$

We finally turn to the polar convexity of the different versions of around,

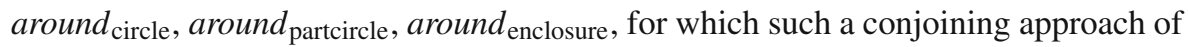
more basic paths is not an option. On the basis of definition (28), the corresponding sets of paths are not necessarily polarly convex. To see this, consider two around $\mathrm{p}_{\text {partcircle }}$ paths $p_{1}$ and $p_{2}$ that have the same constant radius $r$ and the same starting angle $\alpha=0^{\circ}$, but that go in opposite directions (clockwise versus counterclockwise). There is a path $p_{3}$ p-between $p_{1}$ and $p_{2}$ that also has a radius $r$ but that has an angle $\theta$ that is always halfway between the $\theta$ angles of $p_{1}$ and $p_{2}$, that is, the angle $0^{\circ}$. However, this path is not an around $d_{\text {partcircle }}$ path. In fact, it is not even a 'proper' path because it maps only to a single point. See Fig. 17.

The source of the problem is that if two paths go in opposite angular directions then their angles can even each other out in the definition of polar betweenness. A way out of this problem requires a closer look at the directionality of prepositions. What makes route prepositions like around different from goal and source prepositions is that they are 'symmetric'. If a path $p$ is in the set $\operatorname{around}_{\text {partcircle }}(L)$ for a landmark $L$, then so is the path $\sim p$ that is the exact reversal of $p$, i.e., for every $i \in[0,1], \sim p(i)=$ $p(1-i)$. In a sense, within this symmetrical set, $p$ and $\sim p$ are non-distinct paths, because around does not care about the direction of a path. We can say that the paths $p_{1}$ and $p_{2}$ have the same directionality if either $\operatorname{azimuth}\left(p_{1}(0)\right)<\operatorname{azimuth}\left(p_{1}(1)\right)$ and azimuth $\left(p_{2}(0)\right)<\operatorname{azimuth}\left(p_{2}(1)\right)$ or azimuth $\left(p_{1}(0)\right)>\operatorname{azimuth}\left(p_{1}(1)\right)$ and $\operatorname{azimuth}\left(p_{2}(0)\right)>\operatorname{azimuth}\left(p_{2}(1)\right)$. (This definition works for all simple paths, as long as none of the paths cross the line $\theta=0^{\circ}$. In that case, a more complicated definition will become necessary.) As a more precise version of (28), we can then include directionality in the definition of p-convexity of a set of paths $P$ :

(29) A set of paths $P$ is directionally p-convex if and only if, for every path $p_{\mathrm{a}}$ and $p_{\mathrm{c}}$ in $P$ that have the same directionality, and for any path $p_{b}$ that has the same directionality and is p-between $p_{\mathrm{a}}$ and $p_{\mathrm{c}}, p_{b}$ is in $P$.

\footnotetext{
${ }^{14}$ Proof: Suppose that $p_{1}$ and $q_{1}$ from $P$ and $Q$ respectively are conjoined to $p_{1} \wedge q_{1}$ and that $p_{2}$ and $q_{2}$ from $P$ and $Q$ are conjoined to $p_{2} \wedge q_{2}$. Let $p_{3}$ be a path that is polarly between $p_{1}$ and $q_{1}$ and $q_{3}$ a path that is polarly between $p_{2}$ and $q_{2}$ such that $p_{3}(1)=q_{3}(0)$, that is, $p_{3}$ is conjoined to $q_{3} . P$ and $Q$ are convex, so $p_{3}$ is in $P$ and $q_{3}$ is in $Q$. It follows that all points of $p_{3} \wedge q_{3}$ are polarly between $p_{1} \wedge q_{1}$ and $p_{2} \wedge q_{2}$ and hence that $P^{\wedge} Q$ is convex.
} 


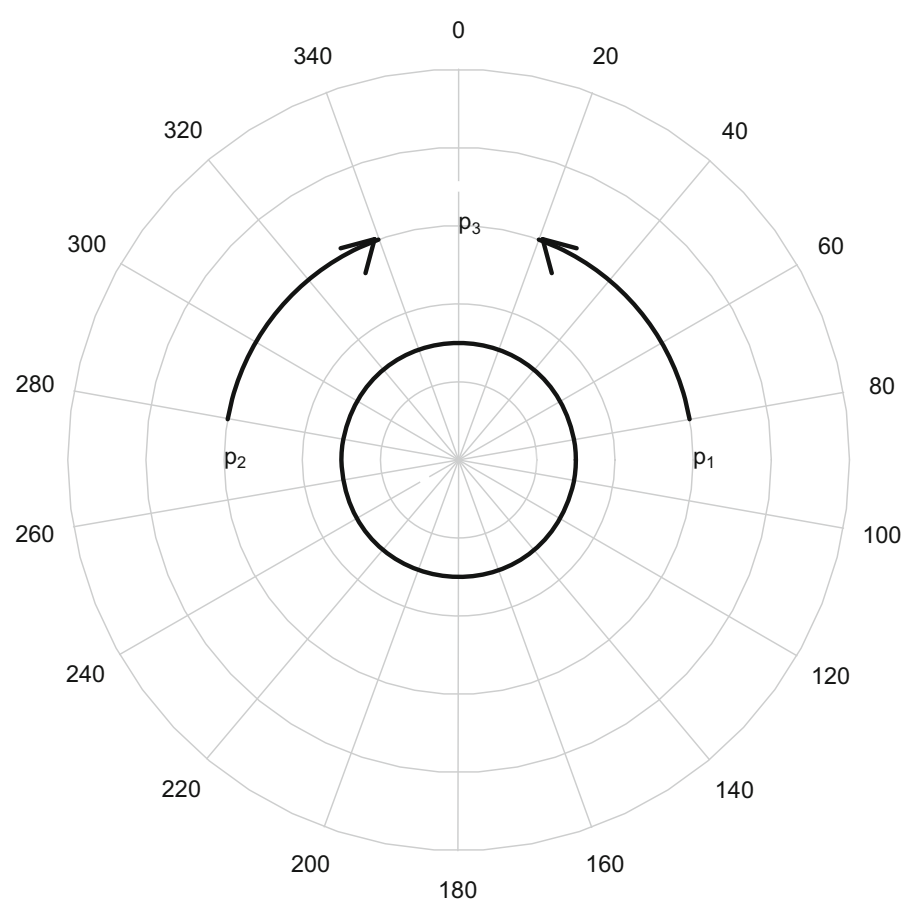

Fig. 17 Non-p-convexity of around paths

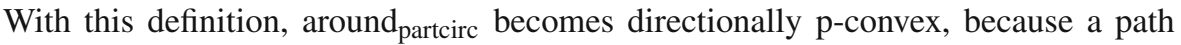
between two others, and with the same direction, will also be a path satisfying the conditions of around partcirc. $_{\text {. }}$

\section{Conclusion}

The main topic of this paper has been the use of geometric notions to describe the semantics of locative and directional prepositions. By using polar coordinates instead of Cartesian coordinates, we have been able to present a unified analysis of most of these prepositions. Locative prepositions denote regions in space and directional prepositions denote simple conditions on paths in space defined in terms of distance (radius) and direction (angle). The use of polar coordinates opens up new computational methods to calculate the meanings of prepositional expressions and new ways to analyze linguistic phenomena within and across languages.

Locative and directional prepositions are prime examples of how meaning is geometrically structured (Gärdenfors 2014). We have been able to show that locative and directional prepositions support the general idea that concepts can be represented by convex regions in conceptual spaces (Gärdenfors 2000), if convexity is defined in terms of a new notion of betweenness over polar coordinates. Polar convexity generalizes and unifies the earlier constraints of continuity in Zwarts (1997). Several prepositions that seem to present counterexamples to polar convexity turn out to have special features. 
The preposition beside is non-convex because of the role of negation, due to competing items, and around required special attention because of its symmetric nature.

Our exploration was based on strong idealizations about the nature and shape of the landmark (as one single, spherical, bounded object referred to by a definite noun phrase). One question for further research is to what extent certain idealizations or schematizations of the shape of a landmark are part of parcel of the way spatial language is used and spatial cognition functions (see Herskovits 1998) and what role convexity plays in those schematizations. Another question is how our approach to convexity extends to landmarks that are plural or that are referred to by indefinite noun phrase. As Mador-Haim and Winter (2015) show, there is an intricate interaction between the properties of the landmark and of the preposition and that interaction also involves convexity.

We set aside some prominent prepositions that cannot be analyzed in purely spatial terms, such as in, on, over, under and against. Some authors (e.g. Coventry et al. 2001; Coventry and Garrod 2004) claim that the semantics of these prepositions have both force dynamic and spatial components. We believe, however, that the spatial component can be derived (metonymically) from the space of forces (see Gärdenfors 2014, Section 11.6-11.8). After all, forces are spatially located. This would also help to explain why some of the force-dynamic prepositions (at, in, on) are the most basic and versatile prepositions used for locating objects and even why they appear as parts of complex prepositions (at the left, in front, on top). It would take us too far, however, to develop this point here.

Since the force fields involved depend to a large extent on the nature of the trajector and the landmark and their relation, it is difficult to determine to what extent the meaning of these prepositions represent convex regions in the force domain or some product space (see Gärdenfors 2014, Sections 11.6-11.8 for a discussion of this). This topic merits further investigation.

Acknowledgments The research for this paper was done during a sabbatical stay of both authors at the Swedish Collegium for Advanced Study, Uppsala, which is hereby gratefully acknowledged. Peter Gärdenfors also gratefully acknowledges support from the Swedish Research Council under the Linnaeus environment Thinking in Time: Cognition, Communication and Learning.

Open Access This article is distributed under the terms of the Creative Commons Attribution 4.0 International License (http://creativecommons.org/licenses/by/4.0/), which permits unrestricted use, distribution, and reproduction in any medium, provided you give appropriate credit to the original author(s) and the source, provide a link to the Creative Commons license, and indicate if changes were made.

\section{References}

Asbury, A., Dotlačil, J., Gehrke, B., \& Nouwen, R. (Eds.). (2008). Syntax and semantics of spatial P. Amsterdam: John Benjamins.

Beliën, M. (2002). Force dynamics in static propositions: Dutch aan, op and tegen. In H. Cuyckens \& G. Radden (Eds.), Perspectives on prepositions (pp. 195-209). Tübingen: Niemeyer.

Bohnemeyer, J. (2012). A vector space semantics for reference frames in Yucatec. In E. Bogal-Allbritten (Ed.), Proceedings of the sixth meeting on the semantics of under-represented languages in the Americas (SULA 6) and SULA-Bar (pp. 15-34). Amherst: GLSA Publications. 
Bowerman, M. (1996). Learning how to structure space for language: A cross-linguistic perspective. In P. Bloom, M. A. Peterson, L. Nadel, \& M. F. Garrett (Eds.), Language and space (pp. 385-436). Cambridge, MA: MIT Press.

Carlson, L., \& van der Zee, E. (Eds.). (2005). Functional features in language and space: Insights from perception, categorization, and development. Oxford: Oxford University Press.

Cohn, A. G., Bennett, B., Gooday, J., \& Gotts, N. N. (1997). Qualitative spatial representation and reasoning with the region connection calculus. Geoinformatica, 1, 275-316.

Cohn, A. G., \& Renz, J. (2008). Qualitative spatial representation and reasoning. In F. van Hermelen, V. Lifschitz, \& B. Porter (Eds.), Handbook of knowledge representation (pp. 551-596). Amsterdam: Elsevier.

Coventry, K. R., \& Garrod, S. C. (2004). Seeing, saying and acting: The psychological semantics of spatial prepositions. Hove, NY: Psychology Press.

Coventry, K. R., Prat-Sala, M., \& Richards, L. (2001). The interplay between geometry and function in the comparison of over, under, above and below. Journal of Memory and Language, 44, 376-398.

Crangle, C., \& Suppes, P. (1989). Geometrical semantics for spatial prepositions. Midwest Studies in Philosophy, 14, 399-422.

Davis, E. (2006). The expressivity of quantifying over regions. Journal of Logic and Computation, 16, 891-916.

De Vries, H. (2015). Shifting sets, hidden atoms: The semantics of distributivity, plurality and animacy. Ph.D. dissertation, Utrecht University.

Dewell, R. (1994). Over again: Image-schema transformations in sematic analysis. Cognitive Linguistics, 5, 351-380.

Eschenbach, C., Tschander, L., Habel, C., \& Kulik, L. (2000). Lexical specifications of paths. In C. Freksa, W. Brauer, C. Habel, \& K. F. Wender (Eds.), Spatial cognition II. Integrating abstract theories, empirical studies, formal methods, and practical applications (pp. 127-144). Berlin: Springer.

Feist, M. I., \& Gentner, D. (1998). On plates, bowls, and dishes: Factors in the use of English IN and ON. In M. A. Gernsbacher \& S. J. Derry (Eds.), Proceedings of the twentieth annual meeting of the cognitive science society (pp. 345-349). Mahwah, NJ: Lawrence Erlbaum Associates.

Gallant, J. L., Braun, J., \& Van Essen, D. C. (1993). Selectivity for polar, hyperbolic, and Cartesian gratings in macaque visual cortex. Science, 259, 100-103.

Gärdenfors, P. (2000). Conceptual spaces: The geometry of thought. Cambridge, MA: MIT Press.

Gärdenfors, P. (2014). Geometry of meaning: Semantics based on conceptual spaces. Cambridge, MA: MIT Press.

Garrod, S. C., Ferrier, G., \& Campbell, S. (1999). 'In' and 'on': Investigating the functional geometry of spatial prepositions. Cognition, 72, 167-189.

Gehrke, B. (2008). Ps in motion: On the semantics and syntax of P elements and motion events. Ph.D. dissertation, Utrecht University.

Habel, C. (1989). Zwischen-Bericht. In C. Habel, M. Herweg, \& K. Rehkämper (Eds.), Raumkonzepte in Verstehensprozessen (pp. 37-69). Tübingen: Niemeyer.

Herskovits, A. (1986). Language and spatial cognition: An interdisciplinary study of the prepositions in English. Cambridge: Cambridge University Press.

Herskovits, A. (1998). Schematization. In P. Olivier \& K. P. Gapp (Eds.), Representation and processing of spatial expressions (pp. 149-162). Mahwah, NJ: Lawrence Erlbaum Associates.

Jackendoff, R. (1983). Semantics and cognition. Cambridge, MA: MIT Press.

Jäger, G. (2010). Natural color categories are convex sets. In M. Aloni \& K. Schulz (Eds.), Amsterdam colloquium 2009 (pp. 11-20). Heidelberg: Springer. LNAI 6042.

Kracht, M. (2002). On the semantics of locatives. Linguistics and Philosophy, 25, 157-232.

Kracht, M. (2008). The fine structure of spatial expressions. In A. Asbury, J. Dotlacil, B. Gehrke, \& R. Nouwen (Eds.), Syntax and semantics of spatial P (pp. 35-62). Amsterdam: John Benjamins.

Landau, B., \& Jackendoff, R. (1993). 'What' and 'where' in spatial language and spatial cognition. Behavioral and Brain Sciences, 16, 217-265.

Leech, G. (1974). Semantics. Harmondsworth: Penguin.

Levinson, S. C. (1996). Frames of reference and Malyneux's question: Cross-linguistic evidence. In P. Bloom, M. A. Peterson, L. Nadel, \& M. F. Garrett (Eds.), Language and space (pp. 109-170). Cambridge, MA: MIT Press.

Levinson, S. C. (2003). Space in language and cognition: Explorations in cognitive diversity. Cambridge: Cambridge University Press. 
Levinson, S. C., \& Wilkins, D. (Eds.). (2006). Grammars of space: Explorations in cognitive diversity. Cambridge: Cambridge University Press.

Lindstromberg, S. (2010). English prepositions explained. Amsterdam: John Benjamins.

Logan, G. D., \& Sadler, D. D. (1996). A computational analysis of the apprehension of spatial relations. In P. Bloom, M. A. Peterson, L. Nadel, \& M. F. Garrett (Eds.), Language and space (pp. 493-529). Cambridge, MA: MIT Press.

Mador-Haim, S., \& Winter, Y. (2015). Far from obvious: The semantics of locative indefinites. Forthcoming in Linguistics and Philosophy.

Mani, I., \& Pustejovsky, J. (2012). Interpreting motion in language. Oxford: Oxford University Press.

Marsden, J. E., \& Tromba, A. J. (1981). Vector calculus (2nd ed.). San Francisco: W.H. Freeman and Company.

Nikitina, T. (2008). Pragmatic factors and variation in the expression of spatial goals: The case of into vs. in. In A. Asbury, J. Dotlačil, B. Gehrke, \& R. Nouwen (Eds.), Syntax and semantics of spatial P (pp. 175-195). Amsterdam: John Benjamins.

O'Keefe, J. (1996). The spatial prepositions in English, vector grammar, and the cognitive map theory. In P. Bloom, M. A. Peterson, L. Nadel, \& M. F. Garrett (Eds.), Language and space (pp. 277-316). Cambridge, MA: MIT Press.

O'Keefe, J. (2003). Vector grammar, places and the functional role of the spatial prepositions in English. In E. van der Zee \& J. Slack (Eds.), Representing direction in language and space (pp. 69-85). Oxford: Oxford University Press.

Pantcheva, M. (2010). The syntactic structure of locations, goals and sources. Linguistics, 48, 1043-1081.

Randell, D. A., Cui, Z., \& Cohn, A. G. (1992). A spatial logic based on region and connection. In Third international conference on knowledge representation and reasoning (pp. 165-176). San Mateo, CA: Morgan Kaufmann.

Regier, T. (1996). The human semantic potential. Spatial language and constrained connectionism. Cambridge, MA: MIT Press.

Regier, T., \& Carlson, L. (2001). Grounding spatial language in perception: An empirical and computational investigation. Journal of Experimental Psychology: General, 130, 273-298.

Saint-Dizier, P. (Ed.). (2006). The Linguistic dimension of prepositions and their use in NLP applications. Dordrecht: Kluwer.

Svenonius, P. (2006). The emergence of axial parts. Nordlyd, 33(1), 49-77.

Svenonius, P. (2008). Projections of P. In A. Asbury, J. Dotlacil, B. Gehrke, \& R. Nouwen (Eds.), Syntax and semantics of spatial $P$ (pp. 63-84). Amsterdam: John Benjamins.

Talmy, L. (2000). Toward a cognitive semantics. Cambridge, MA: MIT Press.

Tyler, A., \& Evans, V. (2001). Reconsidering prepositional polysemy networks: The case of over. Language, 77, 724-765.

Ullman, S. (1984). Visual routines. Cognition, 18, 97-159.

Vandeloise, C. (1991). Spatial prepositions: A case study from French. Chicago, IL: University of Chicago Press.

van der Zee, E., \& Watson, M. (2004). Between space and function: How spatial and functional features determine the comprehension of between. In L. Carlson \& E. van der Zee (Eds.), Functional features in language and space: Insights from perception, categorization and development (pp. 113-127). Oxford: Oxford University Press.

van de Weghe, N., Kuijpers, B., Bogaert, P., \& de Maeyer, Ph. (2005). A qualitative trajectory calculus and the composition of its relations. In M. Rodriguez, I. Cruz, S. Levashkin, \& M. Egenhofer (Eds.), Geospatial semantics, LNCS (Vol. 3799, pp. 60-76). Berlin: Springer.

Vulchanova, M., \& van der Zee, E. (Eds.). (2012). Motion encoding in language and space. Oxford: Oxford University Press.

Wunderlich, D. (1991). How do prepositional phrases fit into compositional syntax and semantics. Linguistics, 29, 591-621.

Zwarts, J. (1997). Vectors as relative positions: A compositional semantics of modified PPs. Journal of Semantics, 14, 57-86.

Zwarts, J. (2003). Paths round a prototype. In P. Saint-Dizier (Ed.), ACL-SIGSEM workshop: The linguistic dimensions of prepositions and their use in computational formalisms and applications (pp. 228-238). Toulouse: IRIT.

Zwarts, J. (2005). Prepositional aspect and the algebra of paths. Linguistics and Philosophy, 28, 739-779. 
Zwarts, J. (2010). Forceful prepositions. In V. Evans \& P. Chilton (Eds.), Language, cognition and space: The state of the art and new directions (pp. 193-214). London: Equinox Publishing.

Zwarts, J., \& Winter, Y. (2000). Vector space semantics: A model-theoretic analysis of locative prepositions. Journal of Logic, Language and Information, 9(2), 171-213. 